\title{
Near-infrared fluorescent probes in cancer imaging and therapy: an emerging field
}

This article was published in the following Dove Press journal:

International Journal of Nanomedicine

5 March 2014

Number of times this article has been viewed

\section{Xiaomin Yi \\ Fuli Wang \\ Weijun Qin \\ Xiaojian Yang \\ Jianlin Yuan}

Department of Urology, Xijing Hospital, Fourth Military Medical University, Xi'an, Shaanxi, People's Republic of China
Correspondence: Jianlin Yuan Department of Urology, Xijing Hospital, Fourth Military Medical University,

I27 Changle West Road, Xi'an,

Shaanxi, People's Republic of China

Tel +86 2984775317

$\mathrm{Fax}+862984775317$

Email yuanjianlin317@|26.com

\begin{abstract}
Near-infrared fluorescence (NIRF) imaging is an attractive modality for early cancer detection with high sensitivity and multi-detection capability. Due to convenient modification by conjugating with moieties of interests, NIRF probes are ideal candidates for cancer targeted imaging. Additionally, the combinatory application of NIRF imaging and other imaging modalities that can delineate anatomical structures extends fluorometric determination of biomedical information. Moreover, nanoparticles loaded with NIRF dyes and anticancer agents contribute to the synergistic management of cancer, which integrates the advantage of imaging and therapeutic functions to achieve the ultimate goal of simultaneous diagnosis and treatment. Appropriate probe design with targeting moieties can retain the original properties of NIRF and pharmacokinetics. In recent years, great efforts have been made to develop new NIRF probes with better photostability and strong fluorescence emission, leading to the discovery of numerous novel NIRF probes with fine photophysical properties. Some of these probes exhibit tumoricidal activities upon light radiation, which holds great promise in photothermal therapy, photodynamic therapy, and photoimmunotherapy. This review aims to provide a timely and concise update on emerging NIRF dyes and multifunctional agents. Their potential uses as agents for cancer specific imaging, lymph node mapping, and therapeutics are included. Recent advances of NIRF dyes in clinical use are also summarized.
\end{abstract}

Keywords: near infrared dyes, nanoparticles, imaging, cancer targeting, cancer therapy

\section{Introduction}

Cancer remains a great challenge against global public health and a tremendous economic burden on society despite significant progress in comprehensive therapy. Recently, the death toll resulting from cancer in the US reached half a million in 2013. It is projected that, in 2014, the total number of newly diagnosed cancer patients will be over 1.6 million in US. ${ }^{1}$ Although interventions such as radical surgical treatment, radiotherapy, and chemotherapy were applied, many patients die within a year after initial cancer diagnosis. The main inadequacy of current diagnostic imaging methods is the relatively low specificity and sensitivity in the detection of cancer at an early stage. Several imaging modalities, such as ultrasound, X-ray radiography, computed tomography $(\mathrm{CT})$, magnetic resonance imaging (MRI), and positron-emission tomography scanning (PETS), are widely utilized to detect the functional and structural changes in disease areas. However, these conventional imaging modalities sometimes fail to achieve a high contrast among malignancies, benign lesions, and adjacent normal tissues. ${ }^{2,3}$ Thus, a novel imaging technique is imperative to enhance the theranostics and improve the surveillance of cancers at any stage. 
Optical imaging holds great promise as an ideal modality for cancer imaging. Light signals emitted from biological tissues present molecular information that is related to the pathophysiological change. Near-infrared fluorescence (NIRF) imaging, with high sensitivity and multi-detection capability, is an attractive modality for early cancer detection among potential optical imaging technologies. This approach basically depends on a fluorescence probe with emissions in the NIR region. In this region (650-900 nm), lower tissue autofluorescence and less fluorescence extinction enhance deep tissue penetration with minimal background interference. $^{4}$

Nanotechnology significantly facilitates the development of both therapeutic and diagnostic agents. Theranostic nanomedicine, the integration between diagnosis and treatment, takes advantage of imaging and therapeutic functions to simultaneously detect and treat disease. Thus, it allows for successive determination of agent distribution, release, and efficacy, which is anticipated to achieve personalized medicine. ${ }^{5-7}$

Strong interest has been attracted in bioimaging and therapeutics of NIRF probes in the last two decades. Numerous novel NIRF dyes with fine photophysical properties have been developed. These dyes can easily be conjugated with various moieties such as small molecules, nucleotides, double-stranded deoxyribonucleic acid (DNA), DNA primers, amino acids, proteins, and antibodies to acquire specific targeting abilities. ${ }^{2}$ Due to convenient modification by conjugating with moieties of interest, NIR dyes are ideal candidates for cancer imaging with high specificity and sensitivity. Nanoparticles (NPs) containing NIRF dyes and anticancer agents contribute to the synergistic management of cancer. Moreover, novel NIRF dyes alone can be utilized as effective agents for photothermal and photodynamic therapy.

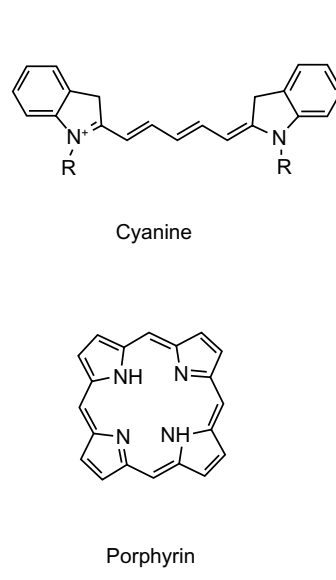

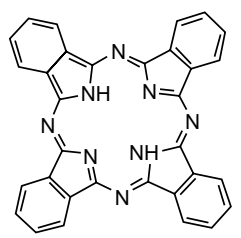

Phthalocyanine

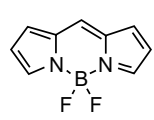

BODIPY

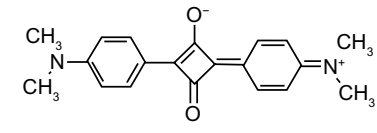

Squaraine

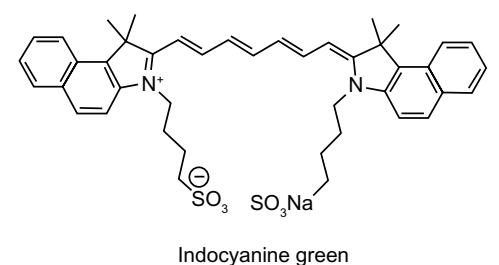

Figure I Basic chemical structures of NIRF dyes.

Abbreviations: NIRF, near infrared fluorescent; BODIPY, 4,4-difluoro-4-bora-3a,4a-diaza-s-indacene. 
to the end of a polymethine that comprises an odd number of carbons. Most of these dyes display narrow absorption bands and high extinction coefficients. Some cyanines (Cy5 and $\mathrm{Cy} 7$ ) emit in the NIR region, presenting as NIRF dyes with relatively strong fluorescence intensity and extinction coefficients. ${ }^{10}$ The elongation of the middle polymethine by the vinylene bond $(\mathrm{CH}=\mathrm{CH})$ results in red shift by approximately $100 \mathrm{~nm}$, and the extension of the nitrogen-containing heterocycles leads to a red shift by $20 \mathrm{~nm} .{ }^{8}$ Traditional cyanine dyes display poor photostability, self-aggregation, and low quantum yields in aqueous solution, while a cyclohexenyl group replacement in the central link of polymethine significantly increases the photostability and fluorescence intensity. ${ }^{2}$ Ongoing efforts in the research of cyanine dyes are focused on exploiting their value in biomedical applications.

James and coworkers constructed a series of symmetrical polymethine dyes containing different bis-N-substituted indoles, linkers, and benzindole moieties. They found that commercially available heptamethine carbocyanine IR-783 with a cyclic chloro-cyclohexene moiety showed better fluorescence-imaging ability. ${ }^{11}$ IR-783 and its derivative MHI-148, have demonstrated potential as optical imaging agents both in vivo and in vitro for the rapid detection of human kidney cancer. ${ }^{12}$ Fluorescent hyaluronan (HA) analogs were developed by linking different molar percentages of IR-783 derivative. Hyaluronidase-mediated HA biodegradation was not influenced by IR-783 conjugation. Moreover, this imaging probe ( $17 \%$ dye in $\mathrm{HA}$ ) could detect HA fragments to study the HA uptake and degradation. ${ }^{13}$ Tan et al developed two multifunctional NIRF heptamethine dyes, IR780 and IR808, with suitable optical characteristics, good biocompatibility, and with the ability to target against cancer cells. ${ }^{14}$ A polyethylene glycol (PEG)ylated IR-786 derivative, synthesized by $\mathrm{Lu}$ et al, indicated a large stokes shift, reduced cytotoxicity, and enhanced photostability. ${ }^{15}$ By functionalization with magnetic NPs and polymers, the stokes shift of IR-820 could be tuned. ${ }^{16}$ New octupolar merocyanine chromophores developed by Poronik et al could also be easily tuned into the NIR region. ${ }^{17}$ The colored conjugate base of 1,3-bis(dicyanomethylidene)indan also displayed the ability as an anionic NIRF dye for biomolecule imaging. ${ }^{18}$

\section{Rhodamine dyes}

Rhodamine dyes belong to the class of xanthene dyes. These dyes have been extensively exploited as fluorescent probes owing to their favorable photophysical characteristics, such as great molar extinction coefficients and resistance in photobleaching. However, the fluorescence probes adapted from classic rhodamine dyes emit visible light only (500-600 nm wavelength), and are thus unavailable for in vivo bioimaging. Interestingly, their fluorescence properties can be easily modified through a ring close/open process or photo-induced electron transfer (PET). ${ }^{19,20}$ Thus, numerous NIR rhodamine derivatives have been developed by modifying the xanthene core, through various mechanisms to regulate fluorescence properties, such as PET, oxidationreduction, and spiro ring opening of xanthenes. ${ }^{8}$

Sun et al revealed three rhodamine derivatives that maintained their properties. They emitted fluorescence in the NIR region with great potential for biological application. ${ }^{21}$ SiR680 and SiR700, synthesized via modifying the Si-rhodamine scaffold, could emit strong NIR fluorescence in aqueous media. ${ }^{22}$ 2-Me TeR, a NIRF dye that selectively probed reactive oxygen species (ROS) utilizing tellurium, could monitor dynamically in vivo endogenous ROS levels. ${ }^{23}$ Unique NIR-absorbing xanthene chromophores could also be synthesized by modulating the HOMO-LUMO (highest occupied molecular orbital-lowest unoccupied molecular orbital) gap in xanthene dyes. ${ }^{24}$

\section{BODIPY-based NIRF probes}

BODIPY, initially depicted in 1968, is advantageous for bioimaging due to high extinction coefficients and quantum yield as well as thermal and photochemical stability. ${ }^{25}$ However, absorption and emission wavelengths of classical BODIPY dyes are not in the NIR region. Therefore, great efforts in seeking ideal BODIPY derivatives have been made by research groups worldwide. Currently, two main strategies are adopted to shift BODIPY into NIRF dyes, modifying the phenyl rings and merging the 3- and 5-phenyl rings with the aza-BODIPY core, which forms six-membered rings and reduces the torsion angles formed by peripheral phenyl groups and the central core. ${ }^{26,27}$ Enhancement of co-planarity between phenyls and the central core results in NIR bathochromic shifts, probably due to electron delocalization. ${ }^{28}$

Diphenyl dithienyl aza-BODIPY was synthesized through the substitution of phenyl groups by thiophene in aza-BODIPY. Remarkable red shifts and strong NIR fluorescence could be found. ${ }^{28}$ Bromo-substituted BODIPY containing thienopyrrole moieties was reported by Yang's group. Exposure of these products in the NIR region exhibited a high singlet oxygen quantum yield that leads to photo cytotoxicity. ${ }^{29}$ Sakamoto and coworkers developed a series of new alpha-bridged linear BODIPY oligomers that displayed strong absorption and high fluorescence efficiency in the NIR region. ${ }^{30}$ Two novel NIRF BODIPY dyes, each containing 
two pyridinium groups showed DNA photocleavage ability through the production of free radicals. These implied the potential mechanism and feasibility of BODIPY-based photodynamic therapy (PDT).${ }^{31}$ DC-SPC, a BODIPY derivative, was fabricated by the classical Knoevenagel condensation method between 3, 5-dimethyl-BODIPY dyes and N-propargyl carbazole aldehyde. DC-SPC emitted in the NIR region and displayed desirable photostability. After facile functionalization into DC-SPC-PPh3, superior amphipathy, cell membrane permeability, and specific localization in mitochondria were revealed. ${ }^{32}$ Stable NIR BODIPY developed via a novel pyrrole was non-cytotoxic and suitable for bio-imaging assay. ${ }^{33}$

\section{Squaraine-based NIRF probes}

Squaraines (squarylium dyes), consisting of a central ringbased core and a zwitterionic structure, were first developed by Treibs and Jacob in $1965 .{ }^{34}$ The oxocyclobutenolate core is linked by aromatic or heterocyclic components at both ends, which shapes a donor-acceptor-donor motif. These dyes typically emit in the red to NIR region, with distinct physical-chemical properties. However, the relatively large scale and hydrophobic features of conventional squaraines remain a grave challenge. Currently, three main strategies for the design of NIRF squaraine dyes are exploited, regulating aggregation or disaggregation of squaraine dyes, modifying squaraine dyes with specific binding moiety, and inducing squaraine formation or destruction in certain conditions. Squaraine-based probes have been developed for different targets, including ions, small molecules, and proteins through these effective strategies. For instance, bis(vinyl ruthenium)-modified squaraine dyes are synthesized through a reversible polyelectrochromic switch that regulates the NIR absorption bands. Fluorescence signal could be easily turned off or shifted deep into the NIR region. ${ }^{35}$ Adding dicyanovinyls into the framework of conventional squaraines enhances NIR fluorescence properties and chemical robustness. ${ }^{36}$

\section{Phthalocyanines and porphyrin derivatives}

Phthalocyanines and porphyrin derivatives are versatile functional pigments containing four isoindole or pyrrole nitrogen atoms. ${ }^{37}$ There are $4 n+2 \pi$ electrons in the middle of phthalocyanines. It was revealed that the $\pi$ electrons are strongly delocalized around the chromophore, which renders high thermal and chemical stability that can endure intense electromagnetic radiation. Moreover, two hydrogen atoms in the central part can be readily modified by metal ions and various substituents, thus changing the physical properties. Although applications in the fields of electronics, optoelectronics, and biomedicine can be found, their emission bands are under the NIR region. ${ }^{2}$ Hence, strategies such as ligating the benzene group and replacing multiple electron-donating substituents can be applied to acquire NIRF derivatives. Porphyrins and expanded porphyrins contain four or more pyrrole or heterocyclic rings. When used as new platforms for NIRF probe design, such macro-cyclic structures show the advantages of superior chemical stability and excellent photophysical properties.

Their new derivatives are also promising in clinical application for cancer theranostics. Karunakaran et al developed a hydrophilic porphyrin (THPP) and its derivative (Zn-THPP). They displayed superior quantum yield and excellent free radical generation rates. In comparison with the clinical drug Photofrin, THPP exhibited higher photodynamic activity. Moreover, THPP rapidly permeated into cells and localized in the nucleus, demonstrating its potential application as a NIR probe for PDT as well as nucleus imaging. ${ }^{38}$

\section{NIRF probes based on other types of dyes}

Besides cyanine dyes, rhodamine derivatives, BODIPYs, porphyrins and squaraines, some other types of NIR fluorophores have also been recently investigated. Ashitate et al demonstrated that zwitterionic NIR fluorophore ZW800-1 could provide realtime intraoperative imaging of hepatic vessels precisely during biliary tract surgery, outperforming ICG in dual-channel NIR fluorescence imaging. ${ }^{39}$ When conjugated with cancer-targeting moieties, ZW800-1 provided a much improved signal-to-noise ratio (SNR) over IRDye800-CW and Cy5.5.40 Hyaluronated fullerene was found to exhibit strong NIRF intensity without complex labelling with other fluorophores or isotopes. This fullerene could be applied for high-resolution NIRF imaging of tumor sites in vivo. Moreover, a remarkable regression of HCT-116 tumors but not normal tissues was found, demonstrating its potential anti-tumor activity. ${ }^{41}$ Xiong et al constructed a NIRF analog of $2^{\prime}, 7^{\prime}$-dichlorofluorescein with high photostability and permeability. This dye was specifically located in the mitochondria and could be readily employed for thiol sensing via a strong turn-on NIRF signal. ${ }^{42}$ Sun et al revealed that two-photon carbazole derivatives could be used as NIRF dyes for nucleic acid imaging applications. ${ }^{43} 2^{\prime}$-hydroxychacone derivatives of boron difluoride were synthesized with intense NIR emission in the solid state. They possessed many interesting properties that were suitable for applications in materials science as unique solid-state NIR fluorophores. ${ }^{44}$ Shao et al reported a new class of NIRF dendrimeric quaterrylenediimide dyes exhibiting high photostability and hydrophilicity 
and low toxicity to normal cells. ${ }^{45}$ A self-quenching composite was constructed by conjugating galactosyl human serum albumin (hGSA) and NMP1, a bacteriochlorin-based NIRF dye. The complex selectively linked to the D-galactose receptor of ovarian cancer cells and $75 \%$ of 555 peritoneal ovarian cancer metastases and was detected by hGSA-NMP1. ${ }^{46}$ Although significant progress in NIRF probe design and their biological applications has been made, they are still fledgling compared to visible light optical imaging and many challenges remain in the development of clinically available NIRF dyes.

\section{NP-based NIRF probes}

Advances in nanotechnology facilitate the development of complex multicomponent nanomaterials, significantly contributing to the fast growing biomedicine branch. Great progress has been made in the field of NP-based probes for cancer theranostics. Newly developed probes selectively accumulate in malignant lesions via the enhanced permeability and retention (EPR) effect of tumor microvasculatures, ${ }^{47,48}$ and bind to specific receptors on cancer cells through corresponding ligands in NPs. Thus, NP-based NIRF probes, such as NIRF dye-containing nonmetallic NPs, NIRF gold nanostructures, and quantum dots (QDs), attract strong interest as platforms for early-stage cancer detection (Figure 2). Three main types of these fascinating probes developed in recent years are described below.

\section{NIRF dye-containing nonmetallic NPs}

NIRF dye-containing nonmetallic NPs are based on nanomaterials of polymer and inorganic matrices that confine various NIRF dyes. Organic NIRF dyes are typically embedded through non-covalent or covalent combination to shape functional NPs with a core-shell architecture. Owing to the protective architecture, these probes show enhanced photostability and biocompatibility, low self-aggregation, bright fluorescence signal, with bioconjugation that is easily tunable. ${ }^{49}$

Different routes to obtain functional NIRF nonmetallic NPs have been employed in recent years. ICG molecules with a polyethylenimine moiety were incorporated into a

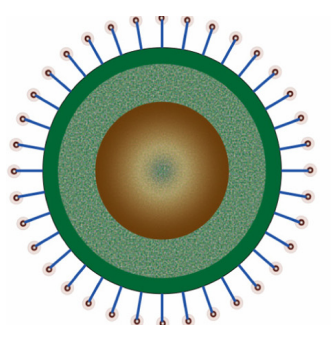

Quantum dots

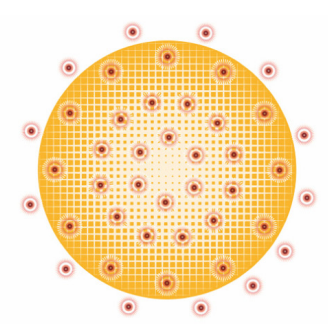

Nonmetallic nanoparticles
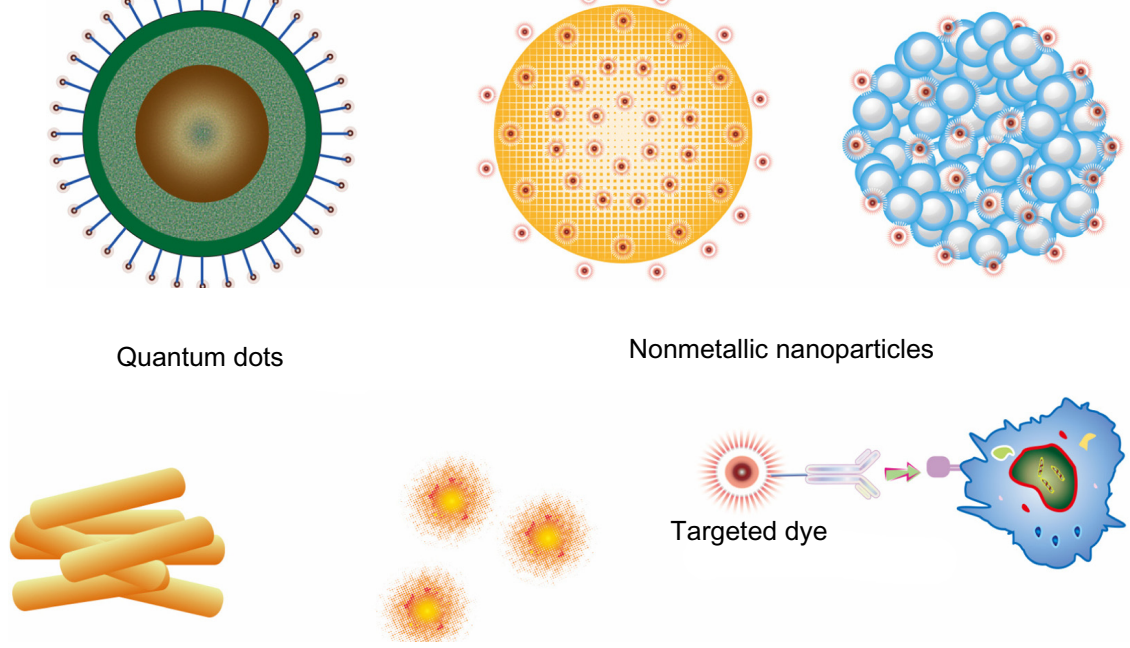

Nanodots

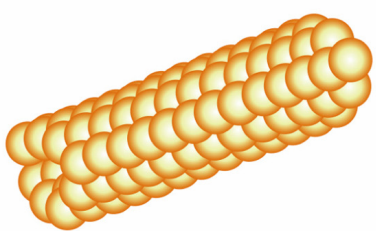

Nanotube

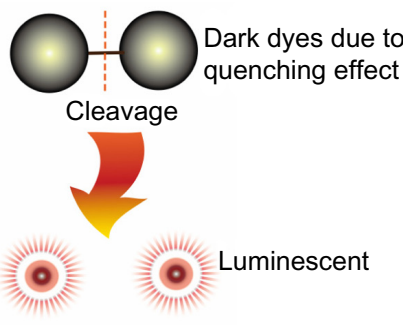

Activatable dyes

Figure 2 Structural characteristics of nanoparticle-based NIRF probes.

Notes: QDs are comprised of a core/shell structure and a coating that minimizes their potential toxicity. Furthermore, specific moieties with targeting ability can be linked to the surface. Nonmetallic nanoparticles include dye-loaded micelles and polymer-based structures that retain NIRF dyes inside or on the surface. Nanorods, nanodots, nanoclusters, and nanotubes are mainly developed using gold nanomaterials. Most of the targeted dyes are linked with moieties that have selective binding activity. Activatable NIRF dyes originally have little fluorescence emission; when disintegrated, these dyes emit strong fluorescence.

Abbreviations: NIRF, near infrared fluorescent; QDs, quantum dots. 
silica matrix, which could avoid aggregation and reduced self-quenching of ICG molecules. ${ }^{50}$ Zheng et al developed a self-assembled ICG-containing nanostructure using ICG and phospholipid-PEG (PL-PEG). ICG-PL-PEG increased the NIR-dependent temperature efficiently and could target tumor cells when further linked with an antibody. ${ }^{51}$ In another study, Cohen et al developed NIRF albumin NPs using human serum albumin (HSA) and a NIR dye derivative in aqueous solution. ${ }^{52}$ The HSA-CANIR NPs were covalently conjugated with tumor-targeting ligands at the carboxylate site through the carbodiimide activation method. NIRF dye encapsulation within the HSA NPs significantly reduced photobleaching. Moreover, the prepared NIRF probes could specifically target colon cancer, even undistinguishable tumors that could previously only be confirmed by histopathological analysis. 1,1'-dioctadecyltetramethyl indotricarbocyanine iodide was incorporated into micelles to acquire dye-loaded NPs. The prepared NPs demarcated the tumor with high resolution, demonstrating the capability of both optical imaging and photothermal treatment. ${ }^{53}$

Despite recent progress in NIRF dye-containing NPs, the characteristic discrepancies such as absorption, biodistribution, metabolism, and excretion remain high owing to large variations in the physicochemical properties. With the ongoing efforts to adapt their functions and endow multiplex imaging capability, NIRF nonmetallic nanoprobes are expected to play important roles for in vivo optical imaging.

\section{Gold nanostructures}

Numerous nanostructures with different sizes and shapes, such as nanorods, nanodots, nanoclusters, nanoshells, nanotubes, and nanocages, have been extensively investigated as candidates for Raman imaging and photoacoustic imaging (PAI) mainly due to their excellent optical properties, high stability, lower toxicity, and bioconjugation simplicity. ${ }^{54-57}$ Fluorescent gold nanoprobes (GNP), characterized by ultrasmall size, tunable optical properties, and considerable biocompatibility, are available as targeted probes for in vivo optical imaging. ${ }^{58}$

Through the modification on poly-(allylamine hydrochloride) followed by Rose Bengal (RB) molecule conjugation, a gold nanorod (GNR) platform was constructed. The RB-GNR platform exhibited significant NIR absorption and emission, excellent photostability, good biocompatibility, and specific identification of oral cancer cells. ${ }^{59} \mathrm{Au}-$ MPA was constructed by the conjugation of bluish-green fluorescence-containing gold nanoclusters (Au NCs) and a NIR organic dye, MPA, and displayed low toxicity and high affinity to tumor. ${ }^{60}$ In another study, folic acid (FA) was linked to $\mathrm{Au}$ NCs followed by MPA conjugation (Au-FA-MPA). Doxorubicin (DOX), a clinically used anticancer drug, was then conjugated to the folate-conjugated Au NCs. Au-FAMPA selectively targeted folate receptor (FR) positive tumors and Au-FA-DOX displayed high anti-tumor activity due to FR-mediated uptake, supporting the potential use of ligandmodified Au NCs for cancer imaging and targeted therapy. ${ }^{61}$ Au-Met-MPA, which was constructed using Au NCs, methionine (Met), and MPA, also exhibited NIR tumor-targeting capability with low cytotoxicity. ${ }^{62}$

Despite advances in nanotechnology, the strategy of incorporating multiple materials and/or agents into one GNP with optimal properties remains challenging. Intractable problems concerning purity, solubility, stability in physiological environments, and the extreme synthetic process of these probes limit their imaging applications in a larger scope.

\section{Quantum dots}

Semiconductor QDs are fluorescent inorganic core-shell nanocrystals with easily modified emission wavelengths and excellent extinction coefficients. Generally, the fluorescence of QDs depends on the size or shape of the intrinsic nanocrystal. QDs with a relatively large core emit longer wavelengths in the NIR region. Therefore, QDs can be finely adapted via their size and composition to obtain suitable excitation profiles and high absorption coefficients. Furthermore, QDs exhibit large Stokes shifts and can endure photobleaching. ${ }^{63}$ Owing to these features, QDs have been extensively investigated for biomedical applications. ${ }^{64}$ However, in vivo imaging with QDs is challenged by their potential toxicity, relatively large size, and short circulation time. To solve the potential acute and chronic toxicity of cadmium-based NIR QDs, a layer of less toxic coating is often considered in the design of QDs. ${ }^{65,66}$ In addition, the water solubility of QDs is crucial to their biological applications.

Xue et al systematically evaluated four different methods to enhance the water solubility of QDs (via micelles, amphiphilic polymers, nanohydrogels, and water-soluble thiols). It was demonstrated that micelles and thiols best retained the photophysical property of QDs. ${ }^{67,68}$ Oil-soluble NIR PbS QDs entrapped in micelles were also investigated for in vivo tumor imaging. Low cytotoxicity $\mathrm{PbS}$ QDs-loaded SOC (biodegradable micelles) selectively accumulated in cancer tissues through the EPR effect of SOC micelles, making them suitable for the evaluation of nanocarrier biodistribution and tumor-targeting behavior. ${ }^{69}$ After modification 
of the QDs with a coating of silica and PEG, the ultra-stable $\mathrm{PbS}$ QDs were available for sentinel lymph node (SLN) imaging. ${ }^{70} \mathrm{Ag}_{2} \mathrm{~S}$ QDs displayed strong optical signals in deep tissue with high resolution. When functionalized with PEG, the composites were found in the reticuloendothelial system and could be removed through feces without appreciable toxicity. ${ }^{71,72} \mathrm{Ag}_{2} \mathrm{~S}$ QDs terminated with a carboxylic acid group showed strong fluorescence and good photostability that is suitable for future biomedical applications. ${ }^{73}$

Biocompatible core/shell QDs were constructed with $\mathrm{Zn}^{2+}$ ions to address the unexpected blue-shift of $\mathrm{CuInS}_{2}$-based nanocrystals. The prepared QDs coupled with cyclic Arg-GlyAsp (cRGD) peptides emitted stronger signals than ZCIS/ZnS QDs in cancer detection. ${ }^{74} \mathrm{CdTeS}$ QDs coated with N-acetyl1-cysteine (NAC) were synthesized as both bioactive ligands and a sulfur source with excellent optical properties and low toxicity. After folate-PEG decoration of the CdTeS alloyed QDs, the conjugated NIR QDs displayed good biocompatibility, excellent sensitivity, and specificity for optical imaging of tumors overexpressing the folate receptor. ${ }^{75}$ Singh et al reported the functionalization of magnetic NIR QDs. When folic acid was covalently linked to these QDs, they exhibited improved specificity and unique magnetic properties. ${ }^{76}$ In Gao's study, dendron-coated InP/ZnS core/shell NIR QDs featured high stability, low cytotoxicity, the ability of extravasation, and were a suitable size for kidney excretion. ${ }^{77}$ Encapsulation of phosphorothiolated oligonucleotides (5-10 nucleotides) into the QD shell resulted in excellent chemical, photonic, and colloidal stability, high quantum yields, and tunable spectral emission. ${ }^{78}$ Loading QDs into deoxycholic acid-conjugated low-molecular-weight heparin micelles rendered excellent stability. These NPs were absorbed via bile acid transportation and cleared via the urinary system. ${ }^{79}$

It is worth noting that NP-based NIRF probes are not limited to the three types aforementioned. Lanthanide compounds, ${ }^{80}$ for example, can be synthesized by $\mathrm{Yb}(3+)$ chelation, ${ }^{81}$ displaying NIR luminescence. Lanthanum hexaboride NPs are suitable for NIRF application since they are easy to prepare, cheaper than gold nanostructure, and have a distinct property of high photothermal conversion rate. ${ }^{82}$

\section{Multimodal NIRF probes}

Although fluorescence imaging is highly sensitive to tagging a corresponding target and is reliable for molecular imaging, few anatomical details can be acquired under microscopic observation. The combinatory application of NIRF imaging and other imaging modalities that can delineate anatomical structures extends fluorometric determination of biomedical information. Various multimodal imaging schemes are designed for facile combination between NIRF probes and traditional imaging approaches that offer in vivo tumor images with excellent spatial resolution, such as MRI, ${ }^{16,83-86}$ CT, PETS,${ }^{87}$ single photon emission computed tomography (SPECT),${ }^{88}$ PAI, ${ }^{89}$ Raman imaging, ${ }^{90,91}$ etc. The main multimodal strategies are superparamagnetic iron oxide and gadolinium MR contrast conjugated with NIRF dyes or NPs. ${ }^{16,92-94}$ Covalent binding of $\mathrm{Fe}_{3} \mathrm{O}_{4}$ and different NIR dyes, such as $\mathrm{Cy} 5$ and QDs, has been extensively reported. ${ }^{95-97} \mathrm{Xi}$ et al combined NIR dye-labeled magnetic iron oxide NPs with a targeting moiety for urokinase plasminogen activator receptor (UPAR). The NIR830-ATF-IONP combination dramatically enhanced photoacoustic signals in tumors. ${ }^{98} \mathrm{~A}$ rhodamine complex was yielded after $\mathrm{Gd}^{3+}$ chelation. The dual-modality paramagnetic/ NIRF probe was feasible for both MRI and NIRF imaging to trace cell migration, homing, and differentiation in vivo.99 Magnetic NPs with NIRF dyes embedded in the silica could be applied as a PET/MRI/NIRF imaging agent for biomedical imaging as well as cell tracking after being labeled with a radioisotope on NPs. ${ }^{87} \mathrm{PAI}$, a non-invasive imaging modality that allows for combined optical signals and ultrasonography, can provide more details of biological tissues. ${ }^{100}$ Both endogenous molecules and exogenous agents render this optical absorption ability. Jeon et al demonstrated the feasibility of photoacoustic cystography by NIR dyes with low toxicity. ${ }^{101}$ Thus, appropriate multimodal molecular probes can exert synergistic advantages over any single modality alone.

\section{NIRF probes with targeting abilities}

Probes with targeting abilities are critical for specific and sensitive bioimaging. Although several native organic dyes display strong and intrinsic capabilities of tumor targeting, most of the NIRF dyes lack specific targeting properties, limiting further bioimaging application. Therefore, attaching targeting moieties to these dyes or NIRF dye-containing NPs is a crucial element in probe design. At present, frequently used targeting moieties are antibodies, peptides, proteins, aptamers, and small receptor ligands ${ }^{102}$ Excitingly, appropriate probe design with targeting moieties almost retains the original NIRF optical properties as well as pharmacokinetics and in vivo biodistribution.

Three main strategies are involved in designing targeted NIRF probes: single coupling between signaling and targeting moieties, target-activatable probes with a turn-on option, and molecular probes with two different targeting sites. ${ }^{103}$ The interaction between activatable probes and their targets triggers a turn-on effect through oxidation or 
enzymatic cleavage, which disintegrates the probes into fluorescent dyes. In that circumstance, initial dark probes due to quenching effects are activated and restore the ability of fluorescence emission. Besides, NPs can also function as carriers by specific receptor identification and/or EPR effect to avoid the direct conjugation of fluorophores and target sites. Therefore, the development of probes with targeting ability holds great promise in targeted cancer imaging and related therapeutic applications.

\section{Native NIRF dyes with tumor-targeting ability}

Several native NIRF dyes have been demonstrated with unique tumor-targeting capability without conjugation to guiding moieties or incorporation into nanostructures. Currently, heptamethine indocyanine dyes such as IR-780 iodide, IR-783 (Figure 3), MHI-148, and porphyrin derivatives $\mathrm{Pz} 247$, are identified with preferential accumulation in cancer tissues, displaying great advantages over the common fluorescent dyes, like ICG. ${ }^{104}$ Although the mechanism accounting for the specific accumulation and retention of these dyes remains unclear, the implications of mitochondrial membrane potential, organic-anion transporting polypeptides (OATPs), and EPR effect in this process have been illustrated. These natively multifunctional NIRF dyes extend our understanding in tumor-targeted imaging.

\section{NIRF NPs with specific targeting ability}

Most of the NIRF dyes require conjugation with targeting moieties to obtain specific binding abilities. These probes are first applied as fluorescent biosensors for real-time tracing of glucose, ${ }^{105}$ metal ions, and anions. ${ }^{106,107}$ With the rapid development in bioimaging, targeted NIRF probes are introduced for cell labeling and tracking in the physiological conditions ${ }^{108}$ as well as morphological and functional evaluations in cancer cells and interstitial tissues, such as the surveillance of $\mathrm{pH}$ change, ${ }^{109}$ hypoxia state, ${ }^{110,111}$ enzyme activity, ${ }^{112,113}$ apoptosis, and necrosis. ${ }^{114}$ The $\mathrm{pH}$ determination relies on the unique chromophores that are susceptible to protonation and deprotonation. Syntheses of receptor-targeted NIRF probes are based on the different receptor-expressing patterns on the surface of cancer cells. A host of receptors have been exploited as ideal targets for NIRF imaging and cancer therapy, such as integrin receptor, ${ }^{112}$ human epidermal growth factor receptor 2 (HER2) receptor, ${ }^{115}$ folate receptor, ${ }^{116,117}$ transferrin receptor, ${ }^{118}$ translocator protein receptor, ${ }^{119}$ endothelin receptor, ${ }^{120}$ somatostatin receptor, ${ }^{121}$ and gastrin-releasing peptide receptor. ${ }^{122}$ The targeting moieties, targets, and NP platforms that have been reported in recent years are summarized in Table 1.

In fact, receptor-targeted NIRF imaging is definitely not limited to the aforementioned receptors. Other targets, such as CXC chemokine receptor type 4 (CXCR4) and CXCR7, prostate specific membrane antigen, uPA, low-density lipoprotein (LDL) receptors, vasoactive intestinal peptide (VIP) receptors, type I insulin-like growth factor receptor (IGF1R), and estrogen receptors have also been extensively investigated for selective NIRF imaging. Meanwhile, various molecules and proteins that are involved in carcinogenesis and tumor microenvironments are included in the design of NIRF probes with specific targeting abilities. ${ }^{123,124}$ Upconversion materials have also been exploited for targeted cancer cell ablation. ${ }^{125}$
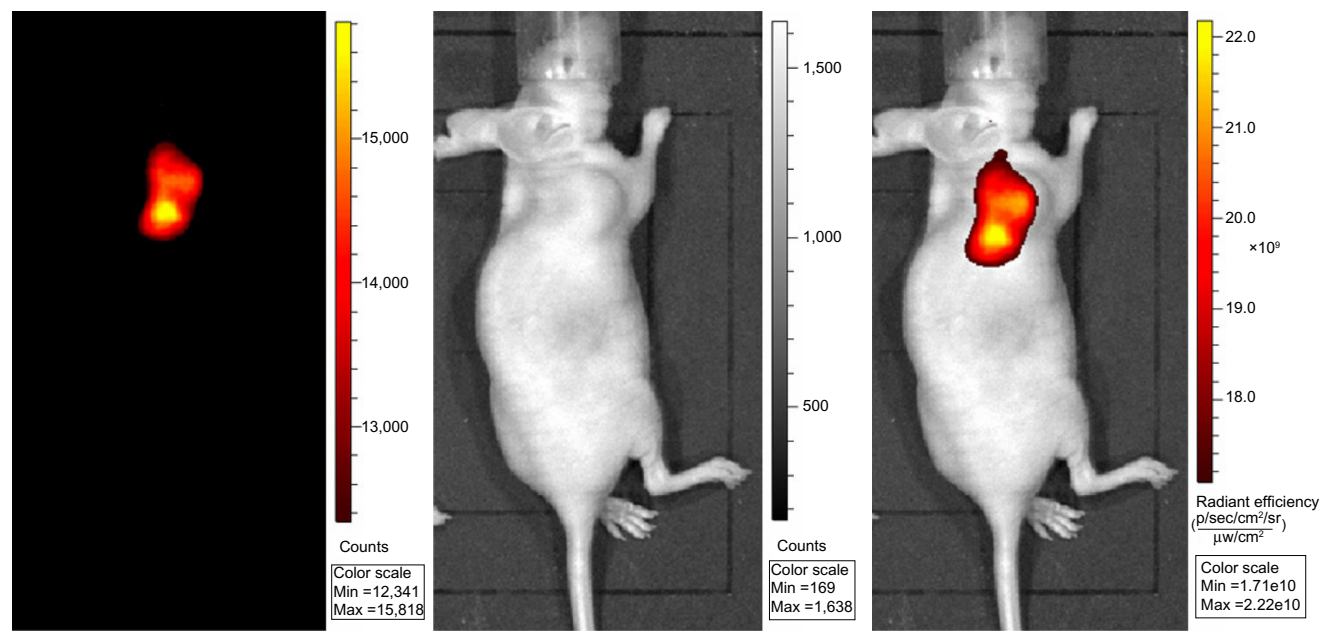

Figure 3 NIRF cancer imaging using IR783 $(0.375 \mathrm{mg} / \mathrm{kg})$ in athymic nude mice with subcutaneous prostate cancer. Abbreviation: min, minimum; max, maximum; NIRF, near infrared fluorescent. 
Table I Targeted NIRF probes

\begin{tabular}{|c|c|c|c|c|}
\hline Targeting agents & Target & NPs or dyes & Linker & $\begin{array}{l}\text { Reference } \\
\text { number }\end{array}$ \\
\hline RGD & Integrin $\alpha v \beta_{3}$ & IRDye $800 \mathrm{CW}$ & Arg-Gly-Asp & 199 \\
\hline RGD & Integrin $\alpha v \beta_{3}$ & ICG-Der-02 & Arg-Gly-Asp & 200 \\
\hline Peptidomimetic antagonist & Integrin $\alpha v \beta_{3}$ & $\begin{array}{l}\text { IntegriSense680 PEG, } \\
\text { IRDye } 800 \mathrm{CW} \text { PEG }\end{array}$ & None & 201 \\
\hline$(R G D) s$ & Integrin $\alpha v \beta_{3}$ & PCLMPs & Arg-Gly-Asp & 202 \\
\hline RGD, octreotate, Ac-TZI400। & Integrin $\alpha v \beta_{3}$ & HAS-Cy5, IR783-CO ${ }_{2} \mathrm{H}$, ICG & Arg-Gly-Asp & 121 \\
\hline EGF & EGFR & Cy5.5 & (NHS) ester & 203 \\
\hline Peptide & EGFR & Cy5.5-PEG & None & 204 \\
\hline ProSense680, MMPSense680 & Cathepsins, MMP & 2DG CW800, EGF CW800 & None & 205 \\
\hline C6 peptide & MMP-2 & Су5.5 & $\mathrm{c}(\mathrm{KAHWGFTLD}) \mathrm{NH}_{2}$ & 206 \\
\hline FA & Folate receptor & ICG-PLGA-lipid NPs & DSPE-PEG2k-FA & 116 \\
\hline Folate & Folate receptor & CdTeS QDs & Folate-PEG conjugated NIR-QDs & 75 \\
\hline FA, DUPA & Folate receptor, PSMA & $\begin{array}{l}\text { Alexa Fluor 647, DyLight 680, } \\
\text { DyLight } 750, \text { IR800CW }\end{array}$ & $\begin{array}{l}\text { Asp-Arg-Asp-Asp-Cys, } \\
\mathrm{HN}\left(\mathrm{CH}_{2}\right)_{2} \mathrm{NH}_{2}\end{array}$ & 117 \\
\hline Lysine-glutamate urea & PSMA & $\begin{array}{l}\text { IRDye800CW, IRDye800RS, } \\
\text { ICG derivative, Cy7, Cy5.5 }\end{array}$ & Lysine-suberate linker & 207 \\
\hline Transferrin & Transferrin receptor & mPEG-b-P(LA-co-MHC/NIR) & None & 118 \\
\hline PKIIII95 & Translocator protein & NIR-conPKIII 95 & None & 119 \\
\hline GXI peptide & Tumor vasculature & Су5.5 & CGNSNPKSC & 120 \\
\hline Bombesin peptides & $\begin{array}{l}\text { Gastrin-releasing peptide } \\
\text { receptor }\end{array}$ & Alexa Fluor 680 & Gly-Gly-Gly & 122 \\
\hline cFLFLF peptide & Formyl peptide receptor & PEG-Oyster-800 & None & 208 \\
\hline Cetuximab & EGFR & 2 SIDCC, 4S-IDCC, 6S-IDCC & None & 209 \\
\hline Trastuzumab & EGFR2 (HER2) & Itrybe & None & 115 \\
\hline
\end{tabular}

Abbreviations: DUPA, 2-(3-[I, 3-dicarboxy propyl]-ureido) pentanedioic acid; EGF, epidermal growth factor; EGFR, epidermal growth factor receptor; FA, folic acid; HER2, human epidermal growth factor receptor 2; ICG, indocyanine green; MMP, matrix metalloproteinases; NHS, N-hydroxysuccinimide; NIR, near-infrared; NP, nanoparticle; PEG, polyethylene glycol; PLGA, poly(lactic-co-glycolic) acid; PSMA, prostate specific membrane antigen; QDs, quantum dots; RGD, Arg-Gly-Asp.

Targeted NIRF technology provides invaluable inspiration for future biomedical application.

\section{Target-activatable NIRF NPs}

Compared with other in vivo bioimaging techniques, NIRF imaging benefits greatly from activatable probes. Specifically, these NPs initially emit little fluorescence owing to self-quenching effects. However, when the probes arrive at the target sites, enzyme cleavage occurs and the fluorophores are released, emitting strong fluorescence. Meanwhile, the non-targeting dark probes cannot be selectively activated to generate fluorescence and are quickly cleared out. Therefore, activatable probes significantly improve the signal-to-noise ratio and specificity in NIRF bioimaging applications. Activatable NIRF signals can be detected even at subnanomolar levels, displaying excellent sensitivity and deep tissue penetration in vivo. Great attention has been paid to the activatable strategy for highly specific targeted imaging. The discrepancies between malignancies and normal tissues, such as the concentrations of specific enzymes, $\mathrm{pH}$ levels, and cellular energy metabolism, were fully exploited to prepare target-activatable NIRF probes. ${ }^{126-128}$ Most probes are special NPs with enzyme cleavable sequences and quenching abilities through two main strategies to control probe fluorescence, PET and Förster resonance energy transfer (FRET), which result in over 99\% quenching in contrast with the free fluorophores. ${ }^{129,130}$ These probes largely depend on the specificity of cleavable sequences used for the coupling of fluorophores and NPs. Numerous enzymes such as matriptase, ${ }^{131}$ protein kinase $\mathrm{C}$ alpha, ${ }^{132} \mathrm{uPA},{ }^{133}$ and matrix metalloproteinase (MMP), ${ }^{134,135}$ have been successfully used to construct activatable NPs, which might also expand to novel probe design utilizing various enzymes and energy donors, as well as energy acceptors. ${ }^{136}$

Other than enzyme activation, selective and sensitive turn-on NIRF probes can be obtained by novel strategies. ${ }^{137}$ Splitting the thiol group of 2,4-dinitrobenzenesulfonyl turns the aza-BODIPY with weak fluorescence to a strong NIR dye that is suitable for the selective detection of cysteine. ${ }^{138}$ $\mathrm{Cy}$-NiSe and Cy-TfSe were constructed as NIRF probes for thiol detection based on the cleavage of the Se-N bond. They exhibited little fluorescence due to the PET process, while glutathione addition gradually increased their fluorescence intensity. ${ }^{139}$ Novel sensors could respond to endogenous thiols or $\mathrm{H}_{2} \mathrm{O}_{2}$ via a turn-on effect, showing the capability of selective NIRF imaging. ${ }^{140}$ Huang et al reported a new 
strategy to detect hydrogen peroxide, glucose, and uric acid using FRET gold NPs, which was in accord with the commercially available method. ${ }^{141}$ NIRF probes could also detect hydrogen sulfide through the cleavage of dinitrophenyl ether, ${ }^{142}$ and carboxylic acid via a switch for fluorescence control by spirocyclization. ${ }^{143}$ These probes appeared to be safe and widely applicable for the noninvasive detection of target biomolecules, making them promising candidates for in vivo imaging applications. ${ }^{144-146}$ These unique strategies for fluorogenic dye design also open new doors for NIRF probe discovery. Activatable NIRF imaging will become a powerful tool for the diagnosis of cancer and other diseases, and a navigation aid during surgery.

\section{NIRF probes in cancer imaging}

Tissues are optically heterogeneous due to different sizes, functional components, and microstructures. This complicates fluorescence imaging due to problems such as autofluorescence, great light absorption, and light scattering. Since the absorption and emission bands of autofluorescence in tissues peak at the near ultraviolet and yellow light regions, NIRF dyes generate less background fluorescence. Moreover, NIRF probes increase the depth of detection in mammalian tissues by several orders of magnitude.

Many NIRF probes have been designed for in vivo cancer imaging applications. Recent development of multifunctional nanomaterials has further improved the availability of NIRF imaging. Nanotechnology-based NIRF probes can efficiently target cancer sites through the EPR effect and selective linking between targeting moieties and tumor cell receptors.

Additionally, NIRF imaging is a potential strategy for intraoperative identification of malignancies. For instance, ICG can clearly identify hepatocellular carcinoma lesions and colorectal cancer liver metastases during surgery. ${ }^{147,148}$ Presently, intraoperative evaluation of tumor margins depends on palpation and visual inspection. NIRF probes with cancertargeting ability can provide instrumental information in demarcating tumors. Thus, complete removal of tumors with adequate tumor-free margins maximizes the benefit of surgery and possibly decreases the risk of recurrence. Sherwinter reported his experience of transanal NIR imaging to assess mucosal perfusion at the level of the anastomoses in patients undergoing colectomy. Intraoperative high-resolution NIR mucosal angiography could be obtained easily, demonstrating the feasibility of transanal NIR angiography in surgery. ${ }^{149}$

Fibroblast activation protein-alpha (FAP- $\alpha)$, a cell surface glycoprotein that promotes tumor growth and invasion, is ubiquitously expressed in malignancies but not in normal tissues. An activatable NIRF probe that contains a NIR dye and a quencher dye was synthesized for real-time imaging of FAP- $\alpha$. This probe selectively accumulated in FAP- $\alpha$ expressing tumors, demonstrating its targeting ability for early cancer detection. ${ }^{150}$

\section{NIRF probes in lymph node imaging}

The peripheral lymphatic system originates from lymphocapillary vessels, where foreign materials, macromolecules, and excess fluid infiltrate out of the blood. The colorless lymph fluid is then transported through collecting vessels, regional lymph nodes, and lymphatic ducts, finally passing back to the blood circulation to maintain tissue fluid homeostasis. Abnormal lymphatic function may be closely related to malignancies and venous disease. ${ }^{151}$ SLNs are the initial draining lymph nodes of tumors. The presence of cancer-positive SLNs usually indicates regional metastases. Therefore, lymph node mapping is a requisite for disease staging, prognosis prediction, and decision making in cancer treatment. The identification and dissection of SLNs using methylene blue dye and radioactive colloid have become the current standard for staging cancers such as breast cancer and melanoma. However, radioactive issues, low spatial resolution, and allergic reactions to the blue dye hamper large-scale application of SLNs mapping. Real-time intraoperative imaging with a high resolution via a flexible, portable non-nuclear modality may facilitate the assessment and resection of the SLNs, and minimize surgical complications.

NIRF dyes have been investigated as a surrogate for radioactive colloid and blue dye imaging. Clear visualization of mammary lymph nodes and lymph vessels by ICG demonstrated the applicability of fluorescence-guided SLN resection. It was further confirmed that ICG and other NIR dyes had little impact on lymphatic function such as propulsive velocity and frequency. ${ }^{152}$ Premixing $150 \mu \mathrm{g} / \mathrm{mL}$ ICG with $60 \mathrm{~g} / \mathrm{L}$ albumin induced greater fluorescence intensity. ${ }^{153}$ In Li's study, QDs were employed in real-time imaging of the rat stomach lymph node basin and functioned well. ${ }^{154}$

Rapid clearance of ICG in the blood limits its use for long-term tracing applications. Therefore, ICG-loaded NPs are widely considered to prolong ICG retention in vivo. Small NPs ( $<50 \mathrm{~nm}$ in diameter) are mainly retained in the lymph nodes, the selective accumulation of small NPs makes it more suitable for lymph node mapping. ${ }^{155} \mathrm{ICG}$ and LI-COR IRDye 800CW PEG were utilized for lymphatic function imaging. The LI-COR tracer remained visible for 2 weeks. Moreover, this dye produced significantly lower effects on lymphatic function than ICG, and lymph nodes were not 
enlarged at any time point, indicating the LI-COR tracer as a more appropriate contrast agent for longitudinal lymphatic imaging. ${ }^{156}$ Jeong et al designed mannosylated liposomeencapsulated ICG (M-LP-ICG) as an optical contrast agent for SLNs mapping. M-LP-ICG had a higher ultraviolet absorbance spectrum, stronger fluorescence intensity, and better stability than LP-ICG, which made it a good contrast agent candidate for the optical imaging of the lymphatic system. ${ }^{157}$ NIR nanogels prepared by the conjugation of IRDye 800 and pullulan-cholesterol polymer nanogels could provide satisfactory imaging of the lymphatic system. ${ }^{158}$

SLNs mapping by NIRF dye have also been implemented in numerous clinical trials. Maus et al investigated NIRF imaging in a lymphedema patient following surgery and radiotherapy to evaluate the lymphatic structure and function. ${ }^{159}$ Ten cancer patients participated in NIRF-imaging-guided dissection of head and neck tumors. Real-time surveillance enabled visualization of SLNs in all ten patients, demonstrating the feasibility of NIRF imaging to detect lymph nodes in patients with head and neck cancer. ${ }^{160}$ Intraoperative SLNs imaging was performed in 25 consecutive patients with biopsy-proven melanoma via NIRF probe (ICG:HSA) and technetium-99m nanocolloid. NIR fluorescent imaging demonstrated an accuracy of $98 \%$ when compared with radioactive colloid. Therefore, SLNs mapping with ICG:HSA is feasible and accurate in melanoma. ${ }^{161}$ Moreover, ICG has the advantage of safety, low cost, and is an intraoperative technique that does not alter the surgical field, thus allowing for easy identification of SLNs. ${ }^{162}$ Stoffels et al investigated intraoperative fluorescence imaging of lymphatic systems by ICG, SPECT/ $\mathrm{CT}$, and preoperative lymphoscintigraphy in 22 patients with melanoma. It was found that 11 additional SLNs were only recognized using fluorescence labeling. ${ }^{163}$ Twenty-two colon cancer patients were included in another study to assess the additional value of NIRF imaging. Ninety-five percent of patients and 77 SLNs were discovered by NIRF dye, compared to 70 SLNs by blue dye. ${ }^{164}$ A hybrid NIRF and radioactive tracer was evaluated for SLN imaging in 32 breast cancer patients. All 48 axillary SLNs were found by radioactive tracer and NIRF dye. ${ }^{165}$ In another study including 49 consecutive breast cancer patients, the use of ICG:HSA also provided additional benefits compared to RC alone. ${ }^{166}$ In 32 women with vulvar cancer, 35 SLNs in 24 patients were intraoperatively detected by NIRF imaging using ICG:HSA and ICG alone, though no significant difference was found between them. ${ }^{167}$ In Rossi et al's study, 17 out of 20 patients with cervical or endometrial carcinoma were observed with SLNs by ICG injection in the cervix, demonstrating that it is a reliable method for intraoperative SLNs mapping. ${ }^{168}$ Eighteen patients with confirmed neoplasia underwent NIR laparoscopy that could transmit both white light and NIR image. Quick switch of NIRF imaging facilitated the confirmation of mesocolic lymphatic drainage patterns and the identification of SLNs. ${ }^{169}$

The aforementioned studies ascertain the effectiveness of NIRF imaging in clinical settings. NIRF imaging has been experiencing explosive development and will be employed in more operating rooms and other biomedical applications. With further progress on multifunctional technologies, there will be more NIRF probes for early cancer imaging, treatment evaluation, and intraoperative tumor delineation, as well as cancer therapy.

\section{NIRF probes in cancer therapy}

In addition to targeted detection of early cancer, NIRF probes exhibit tumoricidal activities at high concentrations. Moreover, cyanine dyes and porphyrin derivatives are potential photosensitizers for photodynamic therapy in cancer treatment. These multifunctional NIRF dyes can be explored for cancer-targeted therapy after further conjugation with specific moieties. For instance, NP-drug formulations can solubilize or shield hydrophobicity or high toxicity to static agents and overcome bioavailability challenges. Further, NP systems can be used to alter pharmacokinetics and increase the percentage of drug accumulation in cancerous lesions. Their favorable size range and the ease of surface functionalization enable targeting of NPs to specific cell types at diseased sites. ${ }^{170}$

\section{Photothermal therapy}

Photothermal therapy (PTT), a noninvasive treatment effective for the treatment of many diseases, has been extensively investigated in cancer treatment. ${ }^{171,172}$ Minimally invasive laser thermal therapy engendered by NPs or drugs, is among the most promising technologies to arrest expansion of cancerous growths with minimal morbidity and reduced toxicity (Figure 4). Laser-absorbing agents or dyes are used to increase laser-induced thermal damage in the tumor.

The accumulation of NIRF probes in tumor sites drastically increases the efficiency of PTT through effective conversion of light energy into heat. ${ }^{82}$ It has been demonstrated that ICG promotes the absorption of NIR laser light delivered by a diode laser, inducing more thermal damage to solid tumors after laser irradiation compared to laser alone. ${ }^{173,174}$ In addition, local hyperthermia 

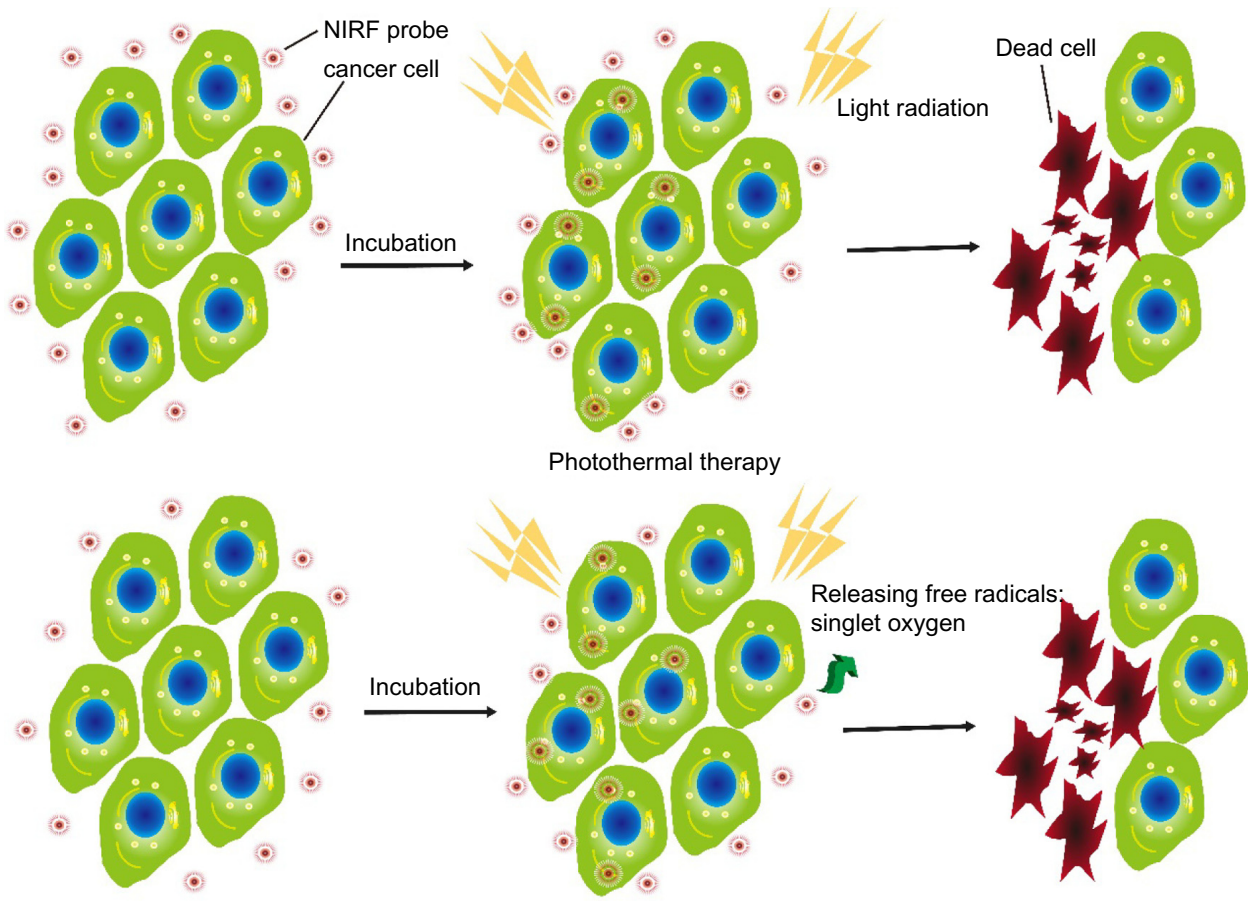

Photothermal therapy

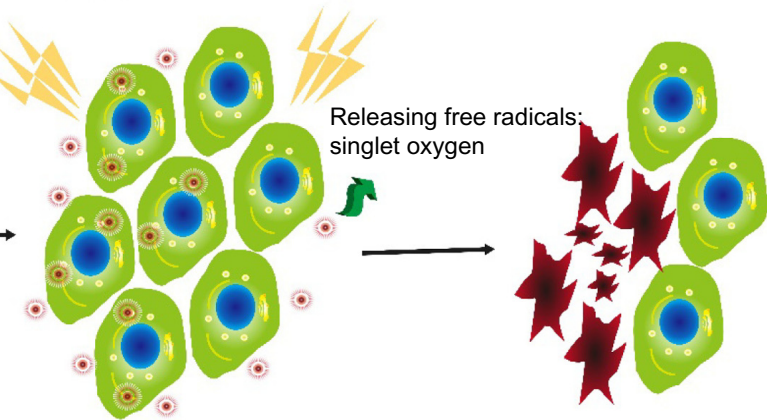

Photodynamic therapy

Figure 4 Schematic illustration of PTT and PDT.

Notes: NIRF probes were incubated with cancer cells. Upon light radiation, the accumulation of NIRF probes drastically increased the efficiency of PTT through effective conversion of light energy into heat, resulting in laser-induced thermal damage to cancer cells. In PDT settings, NIRF probes facilitate the generation of cytotoxicity-free radicals as singlet oxygens, and initiate an inflammatory microenvironment that leads to cancer cell death after light radiation.

Abbreviations: NIRF, near infrared fluorescent; PDT, photodynamic therapy; PTT, photothermal therapy.

greatly enhances the delivery of ICG to the tumor site and interstice, thereby allowing a greater thermal ablation effect of laser therapy on the tumor cells, vasculature, and surrounding tumor matrix to induce tumor regression. Hyperthermia by $\operatorname{ICG}\left(37^{\circ} \mathrm{C}\right.$ to $43^{\circ} \mathrm{C}$ within 1 minute) is also a safe approach to help doxorubicin, a chemotherapy drug, overcome multidrug resistance. ${ }^{175}$ Due to limitations such as poor photostability, self-aggregation, rapid elimination from the body, and lack of target specificity, ICG is usually encapsulated into the core of a polymeric micelle. Interestingly, ICG-encapsulated micelles are still available for potential application in tumor photothermal therapy. ${ }^{51,176}$ Cyanine dye IR820 has optical and thermal generation properties similar to those of ICG but with improved in vitro and in vivo stability. It may be an alternative to ICG with greater stability, longer image collection times, and more predictable peak locations. ${ }^{177}$

Other types of NIRF dyes, such as phthalocyanineaggregated pluronic NPs ${ }^{178}$ and IR780-loaded NPs ${ }^{179,180}$ are also constructed as novel agents for photothermal therapy and/or fractionated photothermal therapy for clinical use. Some of these probes have obtained promising results in preliminary experiments. Researchers will continuously focus on developing suitable NIRF platforms for photothermal applications.

\section{Photodynamic therapy}

PDT utilizes light irradiation that is enhanced by photosensitizers to exert therapeutic effects in cancer tissues. When excited by light at a certain wavelength, photosensitizers facilitate the generation of cytotoxic free radicals (Figure 4$){ }^{3}$ These products affect tumor growth by destroying the abnormal neovasculature directly. They also initiate an inflammatory microenvironment that leads to cancer cell death. ${ }^{181}$ The first approved photosensitizer, Photofrin, is a composite of oligomeric porphyrins that has been applied for the treatment of lung cancer, esophagus cancer, and other types of cancer. In 1993, Photofrin was applied for the first time in PDT to treat bladder tumors. It is noteworthy that current PDT using Photofrin exhibits many drawbacks that limit wide clinical application, such as low deep-tissue penetration, limited tumor specificity, and unwanted localization, especially in the skin, which leads to skin photosensitivity after sunlight exposure. Therefore, developing new photosensitizers without these limitations is imperative for PDT application.

Various NIRF sensitizers have been tested for their use in PDT, such as classical cyanines, squaraines, porphyrins, and phthalocyanines and their derivatives ${ }^{85}$ Precise and real-time demarcation of tumors provided by NIRF probes significantly 
increases the therapeutic efficiency of PDT. Gupta et al reported a new method to construct a multifunctional nanoplatform that could incorporate both the NR fluorophore and photosensitizer for NIRF imaging and photodynamic cancer therapy, making an ideal platform for simultaneous cancer imaging and therapy. ${ }^{182}$ Karunakaran et al developed two hydrophilic porphyrins, THPP and Zn-THPP. In comparison with Photofrin, these probes have stronger intense fluorescence emission and singlet oxygen generation efficiency, showing the potential for PDT. ${ }^{38}$

\section{Photoimmunotherapy}

Photoimmunotherapy (PIT) is based on cancer-targeted therapy that can selectively monitor and destroy cancer tissues. Nakajima et al developed a NIRF probe for PIT by linking a phthalocyanine dye IR700 and a monoclonal antibody. When exposed to NIR light, the conjugates that had been accumulated in the target sites induced highly specific tumoricidal activities. Selective binding avoided unnecessary injury to normal tissues. It was revealed that IR700 eventually accumulated in lysosomes. After exposure to a threshold intensity of NIR light, the conjugates immediately disrupted the outer cell membrane and lysosomes. ${ }^{183,184}$ Furthermore, repeated application of NIRF dyes was an effective strategy for cancer therapy without severe side effects; complete pathological remission might even be achieved. ${ }^{185}$

\section{Drug delivery}

Chemotherapy has been widely applied to maximize the therapeutic outcome in cancer treatment. However, most cytotoxic drugs lack the ability of specific accumulation in tumors. In addition, various side effects may occur during the course of chemotherapy. These remain major impediments to the treatment of malignancies. ${ }^{186}$ Thus, novel platforms for targeted drug delivery that are safe and effective in vivo are highly desirable. Effective delivery of chemical drugs to tumor sites is particularly appealing for the enhancement of the tumor-killing effect and the reduction of systemic toxicities. ${ }^{187,188}$ It was revealed that drug-loaded NP systems accumulate in tumors through the EPR effect, which increases drug bioavailability and prolongs the exposure to therapeutic agents. ${ }^{189}$ Rapid uptake and retention of polymer conjugates in the lymphatic system have also been observed with low toxicity. ${ }^{190}$

Mieszawska et al presented a highly complex and multifunctional hybrid polymer-lipid NP platform that incorporated diagnostic nanocrystals and two therapeutic drugs, the antiangiogenic drug sorafenib and the cytotoxic drug doxorubicin for combined cancer therapy. ${ }^{170}$ The prepared NPs accumulated at the tumor sites and prevented angiogenesis, leading to cancer cell death. NIR irradiation of a light-sensitive amphiphilic copolymer cleaved the cypate-containing micelles and released cytotoxic o-nitrosobenzaldehyde that could damage the surrounding tissues. ${ }^{191}$ Turner et al successfully constructed various temperature-sensitive NIRF mixtures to realize efficient drug delivery. The thermosensitive liposome composites were stable at $37^{\circ} \mathrm{C}$ while burst releases of encapsulated drugs at $40^{\circ} \mathrm{C}$ and $42^{\circ} \mathrm{C}$ were observed. ${ }^{192}$

Currently, antibodies against biomarkers and therapeutic targets of cancer have already been developed. The crosslinking of monoclonal antibodies and NIRF dyes has also been applied for selective cancer theranostics, ${ }^{193}$ such as cutaneous tumor, ${ }^{194,195}$ breast cancer, ${ }^{196}$ ovarian cancer, ${ }^{193}$ gastric cancer, ${ }^{130}$ and prostate cancer. ${ }^{197}$ This controlled drug delivery may potentially address the described limitations of the aforementioned chemotherapy.

\section{Conclusion and perspectives}

Over the last decade, NIRF dyes have been studied extensively for biomedical application since the NIR region is a suitable optical window for deep tissue imaging. When appropriately adapted, these dyes show the abilities of fast screening and early detection of cancer and provide invaluable guidance in cancer therapy, reducing cancer morbidity and mortality. However, conventional dyes experience photobleaching easily and barely achieve sufficient target-to-background ratio for clinical use. Recent developments in nanotechnology and imaging technologies have dramatically facilitated innovations in probe design and multichannel techniques. Many types of NPs have recently been designed and evaluated as potential contrast agents or delivery vehicles for molecular imaging. Successes in the development of receptor-specific NIR nanoprobes and their applications for cancer diagnosis and treatment monitoring have greatly inspired research interest in individualized medical interventions.

NPs provide direct protection of the NIRF dyes against degradation, improve their fluorescence characteristics, and render specific targeting abilities with enhanced signal-tonoise ratio. Although striking results in experimental studies have been obtained, NP-based NIRF probes are still far away from clinical application. ${ }^{198}$ There are still many obstacles before final clinical trials, especially the pharmacokinetics and potential toxicity related to chemical or metallic materials, dosage, and surface coating.

Therefore, non-toxic, biodegradable, and biocompatible materials are highly recommended for constructing the NIR-related probes. Further development of targeted 
NIRF dyes and multimodal probes is expected to broaden their roles in basic cancer research and advance into clinical applications. For rapid progress in this field, interdisciplinary collaboration is still needed.

Taken together, NIRF imaging is promising for early stage cancer detection and cancer therapy, but the development of satisfactory NIRF probes remains challenging for investigators worldwide. Although there are still many hurdles before NIRF imaging can advance to clinical applications, huge opportunities and value exist in this fascinating field.

\section{Acknowledgment}

Funding was provided by the Scientific Innovative Project of Shaanxi Province, No 2012KTCL03-03.

\section{Disclosure}

The authors report no conflicts of interest in this work.

\section{References}

1. Siegel R, Ma J, Zou Z, Jemal A. Cancer statistics, 2014. CA Cancer J Clin. 2014;64(1):9-29.

2. Luo S, Zhang E, Su Y, Cheng T, Shi C. A review of NIR dyes in cancer targeting and imaging. Biomaterials. 2011;32(29):7127-7138.

3. Yuan A, Wu J, Tang X, Zhao L, Xu F, Hu Y. Application of near-infrared dyes for tumor imaging, photothermal, and photodynamic therapies. J Pharm Sci. 2013;102(1):6-28.

4. Wurth C, Hoffmann K, Behnke T, Ohnesorge M, Resch-Genger U. Polymer-and glass-based fluorescence standards for the near infrared (NIR) spectral region. J Fluoresc. 2011;21(3):953-961.

5. McCarthy JR. Multifunctional agents for concurrent imaging and therapy in cardiovascular disease. Adv Drug Deliv Rev. 2010;62(11): 1023-1030.

6. Janib SM, Moses AS, MacKay JA. Imaging and drug delivery using theranostic nanoparticles. Adv Drug Deliv Rev. 2010;62(11):1052-1063.

7. Xie J, Lee S, Chen X. Nanoparticle-based theranostic agents. Adv Drug Deliv Rev. 2010;62(11):1064-1079.

8. Yuan L, Lin W, Zheng K, He L, Huang W. Far-red to near infrared analyteresponsive fluorescent probes based on organic fluorophore platforms for fluorescence imaging. Chem Soc Rev. 2013;42(2):622-661.

9. Barnes KD, Shafirstein G, Webber JS, Koonce NA, Harris Z, Griffin RJ. Hyperthermia-enhanced indocyanine green delivery for laser-induced thermal ablation of carcinomas. Int J Hyperthermia. 2013;29(5): 474-479.

10. Lavis LD, Raines RT. Bright ideas for chemical biology. ACS CHem Biol. 2008;3(3):142-155.

11. James NS, Chen Y, Joshi P, et al. Evaluation of polymethine dyes as potential probes for near infrared fluorescence imaging of tumors: part-1. Theranostics. 2013;3(9):692-702.

12. Yang X, Shao C, Wang R, et al. Optical imaging of kidney cancer with novel near infrared heptamethine carbocyanine fluorescent dyes. J Urol. 2013;189(2):702-710.

13. Wang W, Cameron AG, Ke S. Developing fluorescent hyaluronan analogs for hyaluronan studies. Molecules. 2012;17(2):1520-1534.

14. Tan X, Luo S, Wang D, Su Y, Cheng T, Shi C. A NIR heptamethine dye with intrinsic cancer targeting, imaging and photosensitizing properties. Biomaterials. 2012;33(7):2230-2239.

15. Lu C, Das S, Magut PK, Li M, El-Zahab B, Warner IM. Irradiation induced fluorescence enhancement in PEGylated cyanine-based NIR nano- and mesoscale GUMBOS. Langmuir. 2012;28(40):14415-14423.
16. Yen SK, Janczewski D, Lakshmi JL, et al. Design and synthesis of polymer-functionalized NIR fluorescent dyes - magnetic nanoparticles for bioimaging. ACS Nano. 2013;7(8):6796-6805.

17. Poronik YM, Hugues V, Blanchard-Desce M, Gryko DT. Octupolar merocyanine dyes: a new class of nonlinear optical chromophores. Chemistry. 2012;18(30):9258-9266.

18. Heo J, Lim CK, Whang DR, et al. Self-deprotonation and colorization of 1,3-bis(dicyanomethylidene)indan in polar media: a facile route to a minimal polymethine dye for NIR fluorescence imaging. Chemistry. 2012;18(28):8699-8704.

19. Wang T, Zhao QJ, Hu HG, et al. Spirolactonized Si-rhodamine: a novel NIR fluorophore utilized as a platform to construct Si-rhodamine-based probes. Chem Comm. 2012;48(70):8781-8783.

20. Chen X, Pradhan T, Wang F, Kim JS, Yoon J. Fluorescent chemosensors based on spiroring-opening of xanthenes and related derivatives. Chem Rev. 2012;112(3):1910-1956.

21. Sun YQ, Liu J, Lv X, Liu Y, Zhao Y, Guo W. Rhodamine-inspired farred to near-infrared dyes and their application as fluorescence probes. Angew Chem Int Ed Engl. 2012;51(31):7634-7636.

22. Koide Y, Urano Y, Hanaoka K, et al. Development of NIR fluorescent dyes based on Si-rhodamine for in vivo imaging. J Am Chem Soc. 2012;134(11):5029-5031

23. Koide Y, Kawaguchi M, Urano Y, et al. A reversible near-infrared fluorescence probe for reactive oxygen species based on Te-rhodamine. Chem Comm. 2012;48(25):3091-3093.

24. Sibrian-Vazquez M, Escobedo JO, Lowry M, Fronczek FR, Strongin RM. Field effects induce bathochromic shifts in xanthene dyes. J Am Chem Soc. 2012;134(25):10502-10508.

25. Boens N, Leen V, Dehaen W. Fluorescent indicators based on BODIPY. Chem Soc Rev. 2012;41(3):1130-1172.

26. Zhang T, Zhu X, Wong WK, Tam HL, Wong WY. Light-harvesting ytterbium(III)-porphyrinate-BODIPY conjugates: synthesis, excitationenergy transfer, and two-photon-induced near-infrared-emission studies. Chemistry. 2013;19(2):739-748.

27. Sarma T, Panda PK, Setsune J. Bis-naphthobipyrrolylmethene derived BODIPY complex: an intense near-infrared fluorescent dye. Chem Comm. 2013;49(84):9806-9808.

28. Zhang X, Yu H, Xiao Y. Replacing phenyl ring with thiophene: an approach to longer wavelength aza-dipyrromethene boron difluoride (Aza-BODIPY) dyes. J Org Chem. 2012;77(1):669-673.

29. Yang Y, Guo Q, Chen H, Zhou Z, Guo Z, Shen Z. Thienopyrroleexpanded BODIPY as a potential NIR photosensitizer for photodynamic therapy. Chem Comm. 2013;49(38):3940-3942.

30. Sakamoto N, Ikeda C, Yamamura M, Nabeshima T. alpha-Bridged BODIPY oligomers with switchable near-IR photoproperties by external-stimuli-induced foldamer formation and disruption. Chem Comm. 2012;48(40):4818-4820.

31. Wang J, Hou Y, Lei W, et al. DNA photocleavage by a cationic BODIPY dye through both singlet oxygen and hydroxyl radical: new insight into the photodynamic mechanism of BODIPYs. Chemphyschem. 2012;13(11):2739-2747.

32. Zhang X, Xiao Y, Qi J, et al. Long-wavelength, photostable, two-photon excitable BODIPY fluoro-phores readily modifiable for molecular probes. J Org Chem. 2013;78(18):9153-9160.

33. Jiang XD, Gao R, Yue Y, Sun GT, Zhao W. A NIR BODIPY dye bearing 3,4,4a-trihydroxanthene moieties. Org Biomol Chem. 2012;10(34): 6861-6865.

34. McEwen JJ, Wallace KJ. Squaraine dyes in molecular recognition and self-assembly. Chem Comm. 2009;(42):6339-6351.

35. Chen J, Winter RF. Studies on a vinyl ruthenium-modified squaraine dye: multiple visible/near-infrared absorbance switching through dye- and substituent-based redox processes. Chemistry. 2012;18(34): 10733-10741.

36. Gao FP, Lin YX, Li LL, et al. Supramolecular adducts of squaraine and protein for noninvasive tumor imaging and photothermal therapy in vivo. Biomaterials. 2014;35(3):1004-1014. 
37. Jiang J, Ng DK. A decade journey in the chemistry of sandwich-type tetrapyrrolato-rare Earth complexes. Acc Chem Res. 2009;42(1): 79-88.

38. Karunakaran SC, Babu PS, Madhuri B, et al. In vitro demonstration of apoptosis mediated photodynamic activity and NIR nucleus imaging through a novel porphyrin. ACS Chem Biol. 2013;8(1):127-132.

39. Ashitate Y, Stockdale A, Choi HS, Laurence RG, Frangioni JV. Realtime simultaneous near-infrared fluorescence imaging of bile duct and arterial anatomy. $J$ Surg Res. 2012;176(1):7-13.

40. Choi HS, Gibbs SL, Lee JH, et al. Targeted zwitterionic near-infrared fluorophores for improved optical imaging. Nat Biotechnol. 2013;31(2): 148-153.

41. Kwag DS, Park K, Oh KT, Lee ES. Hyaluronated fullerenes with photoluminescent and antitumoral activity. Chem Comm. 2013;49(3): 282-284.

42. Xiong X, Song F, Chen G, et al. Construction of long-wavelength fluorescein analogues and their application as fluorescent probes. Chemistry. 2013;19(21):6538-6545.

43. Sun Y, Zhao Y, Liu XT, Ren AM, Feng JK, Yu XQ. Theoretical investigation of the two-photon absorption properties of 3,6-bis(4vinylpyridinium) carbazole derivatives - new biological fluorescent probes. J Mol Model. 2012;18(6):2357-2367.

44. D'Aleo A, Gachet D, Heresanu V, Giorgi M, Fages F. Efficient NIRlight emission from solid-state complexes of boron difluoride with 2 'hydroxychalcone derivatives. Chemistry. 2012;18(40):12764-12772.

45. Shao P, Bai M. Photostable, hydrophilic and functional near infrared quaterrylenediimide-cored dendrimers for biomedical imaging. Chem Comm. 2012;48(76):9498-9500.

46. Alexander VM, Sano K, Yu Z, et al. Galactosyl human serum albuminNMP1 conjugate: a near infrared (NIR)-activatable fluorescence imaging agent to detect peritoneal ovarian cancer metastases. Bioconjug Chem. 2012;23(8):1671-1679.

47. Greish K. Enhanced permeability and retention (EPR) effect for anticancer nanomedicine drug targeting. Methods Mol Biol. 2010;624: 25-37.

48. Maeda H. Macromolecular therapeutics in cancer treatment: the EPR effect and beyond. J Control Release. 2012;164(2):138-144.

49. Pellach M, Grinberg I, Margel S. Near IR fluorescent polystyrene/ albumin core/shell nanoparticles for specific targeting of colonic neoplasms. Macromol Biosci. 2012;12(11):1472-1479.

50. Quan B, Choi K, Kim YH, Kang KW, Chung DS. Near infrared dye indocyanine green doped silica nanoparticles for biological imaging. Talanta. 2012;99:387-393.

51. Zheng X, Zhou F, Wu B, Chen WR, Xing D. Enhanced tumor treatment using biofunctional indocyanine green-containing nanostructure by intratumoral or intravenous injection. Mol Pharm. 2012;9(3):514-522.

52. Cohen S, Margel S. Engineering of near IR fluorescent albumin nanoparticles for in vivo detection of colon cancer. J Nanobiotechnol 2012;10:36.

53. Shan G, Weissleder R, Hilderbrand SA. Upconverting organic dye doped core-shell nano-composites for dual-modality NIR imaging and photo-thermal therapy. Theranostics. 2013;3(4):267-274.

54. Zhang X, Bloch S, Akers W, Achilefu S. Near-infrared molecular probes for in vivo imaging. Curr Protoc Cytom. 2012; Chapter 12:Unit12.27.

55. Tian L, Gandra N, Singamaneni S. Monitoring controlled release of payload from gold nanocages using surface enhanced Raman scattering. ACS Nano. 2013;7(5):4252-4260.

56. Raut S, Rich R, Fudala R, et al. Resonance energy transfer between fluorescent BSA protected Au nanoclusters and organic fluorophores. Nanoscale. 2013;6(1):385-391.

57. Antaris AL, Robinson JT, Yaghi OK, et al. Ultra-low doses of chirality sorted $(6,5)$ carbon nanotubes for simultaneous tumor imaging and photothermal therapy. ACS Nano. 2013;7(4):3644-3652.

58. Wang J, Moore J, Laulhe S, Nantz M, Achilefu S, Kang KA. Fluorophore-gold nanoparticle complex for sensitive optical biosensing and imaging. Nanotechnology. 2012;23(9):095501.
59. Wang JH, Wang B, Liu Q, et al. Bimodal optical diagnostics of oral cancer based on Rose Bengal conjugated gold nanorod platform. Biomaterials. 2013;34(17):4274-4283.

60. Chen H, Li B, Wang C, et al. Characterization of a fluorescence probe based on gold nanoclusters for cell and animal imaging. Nanotechnology. 2013;24(5):055704.

61. Chen H, Li S, Li B, et al. Folate-modified gold nanoclusters as near-infrared fluorescent probes for tumor imaging and therapy. Nanoscale. 2012;4(19):6050-6064.

62. Chen H, Li B, Ren X, et al. Multifunctional near-infrared-emitting nano-conjugates based on gold clusters for tumor imaging and therapy. Biomaterials. 2012;33(33):8461-8476.

63. Lu Y, Su Y, Zhou Y, et al. In vivo behavior of near infrared-emitting quantum dots. Biomaterials. 2013;34(17):4302-4308.

64. Kawabori M, Kuroda S, Sugiyama T, et al. Intracerebral, but not intravenous, transplantation of bone marrow stromal cells enhances functional recovery in rat cerebral infarct: an optical imaging study. Neuropathology. 2012;32(3):217-226.

65. Helle M, Cassette E, Bezdetnaya L, et al. Visualisation of sentinel lymph node with indium-based near infrared emitting Quantum Dots in a murine metastatic breast cancer model. PloS one. 2012;7(8):e44433.

66. Gu YP, Cui R, Zhang ZL, Xie ZX, Pang DW. Ultrasmall near-infrared $\mathrm{Ag} 2 \mathrm{Se}$ quantum dots with tunable fluorescence for in vivo imaging. J Am Chem Soc. 2012;134(1):79-82.

67. Xue B, Cao J, Deng D, et al. Four strategies for water transfer of oilsoluble near-infrared-emitting PbS quantum dots. J Mater Sci Mater Med. 2012;23(3):723-732.

68. Deng D, Xia J, Cao J, et al. Forming highly fluorescent near-infrared emitting $\mathrm{PbS}$ quantum dots in water using glutathione as surfacemodifying molecule. J Colloid Interface Sci. 2012;367(1):234-240.

69. Cao J, Zhu H, Deng D, et al. In vivo NIR imaging with PbS quantum dots entrapped in biodegradable micelles. J Biomed Mater Res A. 2012;100(4):958-968.

70. Wang D, Qian J, Cai F, He S, Han S, Mu Y. 'Green'-synthesized near-infrared $\mathrm{PbS}$ quantum dots with silica-PEG dual-layer coating: ultrastable and biocompatible optical probes for in vivo animal imaging. Nanotechnology. 2012;23(24):245701.

71. Zhang Y, Hong G, Zhang Y, et al. Ag2S quantum dot: a bright and biocompatible fluorescent nanoprobe in the second near-infrared window. ACS nano. 2012;6(5):3695-3702.

72. Zhang Y, Zhang Y, Hong G, et al. Biodistribution, pharmacokinetics and toxicology of $\mathrm{Ag} 2 \mathrm{~S}$ near-infrared quantum dots in mice. Biomaterials. 2013;34(14):3639-3646.

73. Jiang P, Zhu CN, Zhang ZL, Tian ZQ, Pang DW. Water-soluble $\mathrm{Ag}(2) \mathrm{S}$ quantum dots for near-infrared fluorescence imaging in vivo. Biomaterials. 2012;33(20):5130-5135.

74. Guo W, Chen N, Tu Y, et al. Synthesis of Zn-Cu-In-S/ZnS core/ shell quantum dots with inhibited blue-shift photoluminescence and applications for tumor targeted bioimaging. Theranostics. 2013;3(2): 99-108.

75. Xue B, Deng DW, Cao J, et al. Synthesis of NAC capped near infraredemitting CdTeS alloyed quantum dots and application for in vivo early tumor imaging. Dalton Trans. 2012;41(16):4935-4947.

76. Singh N, Charan S, Sanjiv K, et al. Synthesis of tunable and multifunctional Ni-doped near-infrared QDs for cancer cell targeting and cellular sorting. Bioconjug Chem. 2012;23(3):421-430.

77. Gao J, Chen K, Luong R, et al. A novel clinically translatable fluorescent nanoparticle for targeted molecular imaging of tumors in living subjects. Nano Lett. 2012;12(1):281-286.

78. Deng Z, Samanta A, Nangreave J, Yan H, Liu Y. Robust DNAfunctionalized core/shell quantum dots with fluorescent emission spanning from UV-vis to near-IR and compatible with DNA-directed self-assembly. J Am Chem Soc. 2012;134(42):17424-17427.

79. Khatun Z, Nurunnabi M, Cho KJ, Lee YK. Oral delivery of near-infrared quantum dot loaded micelles for noninvasive biomedical imaging. ACS Appl Mater Interfaces. 2012;4(8):3880-3887. 
80. Menelaou M, Ouharrou F, Rodriguez L, Roubeau O, Teat SJ, AliagaAlcalde N. Dy(III)- and Yb(III)-curcuminoid compounds: original fluorescent single-ion magnet and magnetic near-IR luminescent species. Chemistry. 2012;18(37):11545-11549.

81. Terai T, Urano Y, Izumi S, Kojima H, Nagano T. A practical strategy to create near-infrared luminescent probes: conversion from fluoresceinbased sensors. Chem Comm. 2012;48(23):2840-2842.

82. Lai BH, Chen DH. LaB6 nanoparticles with carbon-doped silica coating for fluorescence imaging and near-IR photothermal therapy of cancer cells. Acta Biomater. 2013;9(7):7556-7563.

83. Ni D, Zhang J, Bu W, et al. Dual-targeting upconversion nanoprobes across the blood-brain barrier for magnetic resonance/fluorescence imaging of intracranial glioblastoma. ACS Nano. 2014;8(2):1231-1242.

84. Yeh CS, Su CH, Ho WY, et al. Tumor targeting and MR imaging with lipophilic cyanine-mediated near-infrared responsive porous Gd silicate nanoparticles. Biomaterials. 2013;34(22):5677-5688.

85. Qiao XF, Zhou JC, Xiao JW, Wang YF, Sun LD, Yan CH. Triplefunctional core-shell structured upconversion luminescent nanoparticles covalently grafted with photosensitizer for luminescent, magnetic resonance imaging and photodynamic therapy in vitro. Nanoscale. 2012;4(15):4611-4623.

86. Chen H, Wang Z, Ma X, Zong S, Cui Y. Magnetically controllable dual-mode nanoprobes for cell imaging with an onion-liked structure. Talanta. 2013;116:978-984.

87. Kim JS, Kim YH, Kim JH, et al. Development and in vivo imaging of a PET/MRI nanoprobe with enhanced NIR fluorescence by dye encapsulation. Nanomedicine. 2012;7(2):219-229.

88. Guo Y, Yuan H, Cho H, et al. High efficiency diffusion molecular retention tumor targeting. PloS one. 2013;8(3):e58290.

89. Pan D, Cai X, Kim B, Stacy AJ, Wang LV, Lanza GM. Rapid synthesis of near infrared polymeric micelles for real-time sentinel lymph node imaging. Adv Healthc Mater. 2012;1(5):582-589.

90. Satpathy M, Wang L, Zielinski R, et al. Active targeting using HER-2affibody-conjugated nanoparticles enabled sensitive and specific imaging of orthotopic HER-2 positive ovarian tumors. Small. Epub August 27, 2013.

91. O’Hanlon CE, Amede KG, O’Hear MR, Janjic JM. NIR-labeled perfluoropolyether nanoemulsions for drug delivery and imaging. J Fluor Chem. 2012;137:27-33.

92. Sharma P, Bengtsson NE, Walter GA, et al. Gadolinium-doped silica nanoparticles encapsulating indocyanine green for near infrared and magnetic resonance imaging. Small. 2012;8(18):2856-2868.

93. Wei X, Wang W, Chen K. Preparation and characterization of ZnS:Tb, Gd and $\mathrm{ZnS}$ :Er,Yb,Gd nanoparticles for bimodal magnetic-fluorescent imaging. Dalton Trans. 2013;42(5):1752-1759.

94. Ma Y, Tong S, Bao G, Gao C, Dai Z. Indocyanine green loaded SPIO nanoparticles with phospholipid-PEG coating for dual-modal imaging and photothermal therapy. Biomaterials. 2013;34(31):7706-7714.

95. Wate PS, Banerjee SS, Jalota-Badhwar A, et al. Cellular imaging using biocompatible dendrimer-functionalized graphene oxidebased fluorescent probe anchored with magnetic nanoparticles. Nanotechnology. 2012;23(41):415101.

96. Li J, Jiang H, Yu Z, et al. Multifunctional uniform core-shell Fe3O4@mSiO2 mesoporous nanoparticles for bimodal imaging and photothermal therapy. Chem Asian J. 2013;8(2):385-391.

97. Ma Q, Nakane Y, Mori Y, et al. Multilayered, core/shell nanoprobes based on magnetic ferric oxide particles and quantum dots for multimodality imaging of breast cancer tumors. Biomaterials. 2012;33(33): 8486-8494.

98. Xi L, Grobmyer SR, Zhou G, Qian W, Yang L, Jiang H. Molecular photoacoustic tomography of breast cancer using receptor targeted magnetic iron oxide nanoparticles as contrast agents. J Biophotonics. Epub November 2, 2012.

99. Wang XY, Ju S, Li C, et al. Non-invasive imaging of endothelial progenitor cells in tumor neovascularization using a novel dualmodality paramagnetic/near-infrared fluorescence probe. PloS one. 2012;7(11):e50575.
100. Gutrath BS, Beckmann MF, Buchkremer A, et al. Size-dependent multispectral photoacoustic response of solid and hollow gold nanoparticles. Nanotechnology. 2012;23(22):225707.

101. Jeon M, Kim J, Kim C. Photoacoustic cystography. J Vis Exp. 2013;(76):e50340.

102. Cheng K, Cheng Z. Near infrared receptor-targeted nanoprobes for early diagnosis of cancers. Curr Med Chem. 2012;19(28):4767-4785.

103. Bai M, Bornhop DJ. Recent advances in receptor-targeted fluorescent probes for in vivo cancer imaging. Curr Med Chem. 2012;19(28): 4742-4758.

104. Yang X, Shi C, Tong R, et al. Near IR heptamethine cyanine dye-mediated cancer imaging. Clin Cancer Res. 2010;16(10):2833-2844.

105. Khan F, Pickup JC. Near-infrared fluorescence glucose sensing based on glucose/galactose-binding protein coupled to 651-Blue Oxazine. Biochem Biophys Res Comm. 2013;438(3):488-492.

106. Kar C, Adhikari MD, Ramesh A, Das G. NIR- and FRET-based sensing of $\mathrm{Cu} 2+$ and $\mathrm{S} 2-$ in physiological conditions and in live cells. Inorg Chem. 2013;52(2):743-752.

107. Zhu W, Huang X, Guo Z, Wu X, Yu H, Tian H. A novel NIR fluorescent turn-on sensor for the detection of pyrophosphate anion in complete water system. Chem Comm. 2012;48(12):1784-1786.

108. Armentero MT, Bossolasco P, Cova L. Labeling and tracking of human mesenchymal stem cells using near-infrared technology. Methods $\mathrm{Mol}$ Biol. 2013;1052:13-28.

109. Fan L, Fu YJ, Liu QL, Lu DT, Dong C, Shuang SM. Novel far-visible and near-infrared $\mathrm{pH}$ probes based on styrylcyanine for imaging intracellular pH in live cells. Chem Comm. 2012;48(91):11202-11204.

110. Okuda K, Okabe Y, Kadonosono T, et al. 2-Nitroimidazoletricarbocyanine conjugate as a near-infrared fluorescent probe for in vivo imaging of tumor hypoxia. Bioconjug Chem. 2012;23(3):324-329.

111. Xu K, Wang F, Pan X, et al. High selectivity imaging of nitroreductase using a near-infrared fluorescence probe in hypoxic tumor. Chem Comm. 2013;49(25):2554-2556.

112. Shen D, Bai M, Tang R, et al. Dual fluorescent molecular substrates selectively report the activation, sustainability and reversibility of cellular PKB/Akt activity. Sci Rep. 2013;3:1697.

113. Cheng TC, Roffler SR, Tzou SC, et al. An activity-based near-infrared glucuronide trapping probe for imaging beta-glucuronidase expression in deep tissues. J Am Chem Soc. 2012;134(6):3103-3110.

114. Thakur ML, Zhang K, Paudyal B, et al. Targeting apoptosis for optical imaging of infection. Mol Imaging Biol. 2012;14(2):163-171.

115. Behnke T, Mathejczyk JE, Brehm R, et al. Target-specific nanoparticles containing a broad band emissive NIR dye for the sensitive detection and characterization of tumor development. Biomaterials. 2013;34(1):160-170.

116. Zheng C, Zheng M, Gong P, et al. Indocyanine green-loaded biodegradable tumor targeting nanoprobes for in vitro and in vivo imaging. Biomaterials. 2012;33(22):5603-5609.

117. Kelderhouse LE, Chelvam V, Wayua C, et al. Development of tumortargeted near infrared probes for fluorescence guided surgery. Bioconju Chem. 2013;24(6):1075-1080.

118. Yue J, Liu S, Wang R, et al. Transferrin-conjugated micelles: enhanced accumulation and antitumor effect for transferrin-receptoroverexpressing cancer models. Mol Pharm. Epub June 6, 2012.

119. Wyatt SK, Manning HC, Bai M, et al. Preclinical molecular imaging of the translocator protein (TSPO) in a metastases model based on breast cancer xenografts propagated in the murine brain. Curr Mol Med. 2012;12(4):458-466.

120. Chen K, Yap LP, Park R, et al. A Cy5.5-labeled phage-displayed peptide probe for near-infrared fluorescence imaging of tumor vasculature in living mice. Amino acids. 2012;42(4):1329-1337.

121. Bunschoten A, Buckle T, Kuil J, et al. Targeted non-covalent selfassembled nanoparticles based on human serum albumin. Biomaterials. 2012;33(3):867-875.

122. Cai QY, Yu P, Besch-Williford C, et al. Near-infrared fluorescence imaging of gastrin releasing peptide receptor targeting in prostate cancer lymph node metastases. Prostate. 2013;73(8):842-854. 
123. Wu P, Gao Y, Zhang H, Cai C. Aptamer-guided silver-gold bimetallic nanostructures with highly active surface-enhanced Raman scattering for specific detection and near-infrared photothermal therapy of human breast cancer cells. Anal Chem. 2012;84(18):7692-7699.

124. Suganami A, Toyota T, Okazaki S, et al. Preparation and characterization of phospholipid-conjugated indocyanine green as a near-infrared probe. Bioorg Med Chem Lett. 2012;22(24):7481-7485.

125. Xu QC, Zhang Y, Tan MJ, et al. Anti-cAngptl4 Ab-conjugated $\mathrm{N}-\mathrm{TiO}(2) / \mathrm{NaYF}(4): \mathrm{Yb}, \mathrm{Tm}$ nanocomposite for near infrared-triggered drug release and enhanced targeted cancer cell ablation. Adv Healthc Mater. 2012;1(4):470-474.

126. Guo J, Du C, Shan L, et al. Comparison of near-infrared fluorescent deoxyglucose probes with different dyes for tumor diagnosis in vivo. Contrast Media Mol Imaging. 2012;7(3):289-301.

127. Wang Z, Zhang X, Huang P, et al. Dual-factor triggered fluorogenic nanoprobe for ultrahigh contrast and subdiffraction fluorescence imaging. Biomaterials. 2013;34(26):6194-6201.

128. Sun C, Wang P, Li L, et al. A new near-infrared neutral $\mathrm{pH}$ fluorescent probe for monitoring minor $\mathrm{pH}$ changes and its application in imaging of HepG2 cells. Appl Biochem Biotech. Epub October 19, 2013.

129. He X, Wang Y, Wang K, Chen M, Chen S. Fluorescence resonance energy transfer mediated large stokes shifting near-infrared fluorescent silica nanoparticles for in vivo small-animal imaging. Anal Chem. 2012;84(21):9056-9064.

130. Shimizu Y, Temma T, Hara I, et al. Micelle-based activatable probe for in vivo near-infrared optical imaging of cancer biomolecules. Nanomedicine. 2014;10(1):187-195.

131. Deng D, Zhang D, Li Y, Achilefu S, Gu Y. Gold nanoparticles based molecular beacons for in vitro and in vivo detection of the matriptase expression on tumor. Biosens Bioelectron. 2013;49:216-221.

132. Toita R, Mori T, Naritomi Y, et al. Fluorometric detection of protein kinase Calpha activity based on phosphorylation-induced dissociation of a polyion complex. Anal Biochem. 2012;424(2):130-136.

133. Wang J, Wheeler D, Zhang JZ, Achilefu S, Kang KA. NIR fluorophore-hollow gold nanosphere complex for cancer enzymetriggered detection and hyperthermia. Adv Exp Med Biol. 2013;765: 323-328.

134. Myochin T, Hanaoka K, Komatsu T, Terai T, Nagano T. Design strategy for a near-infrared fluorescence probe for matrix metalloproteinase utilizing highly cell permeable boron dipyrromethene. J Am Chem Soc. 2012;134(33):13730-13737.

135. Akers WJ, Xu B, Lee H, et al. Detection of MMP-2 and MMP-9 activity in vivo with a triple-helical peptide optical probe. Bioconjug Chem. 2012;23(3):656-663.

136. Huang X, Lan T, Zhang B, Ren J. Gold nanoparticle-enzyme conjugates based FRET for highly sensitive determination of hydrogen peroxide, glucose and uric acid using tyramide reaction. Analyst. 2012;137(16):3659-3666.

137. Maity D, Govindaraju T. A turn-on NIR fluorescence and colourimetric cyanine probe for monitoring the thiol content in serum and the glutathione reductase assisted glutathione redox process. Org Biomol Chem. 2013;11(13):2098-2104.

138. Jiang XD, Zhang J, Shao X, Zhao W. A selective fluorescent turn-on NIR probe for cysteine. Org Biomol Chem. 2012;10(10):1966-1968.

139. Wang R, Chen L, Liu P, Zhang Q, Wang Y. Sensitive near-infrared fluorescent probes for thiols based on Se-N bond cleavage: imaging in living cells and tissues. Chemistry. 2012;18(36):11343-11349.

140. Yuan L, Lin W, Zhao S, et al. A unique approach to development of near-infrared fluorescent sensors for in vivo imaging. $J$ Am Chem Soc. 2012;134(32):13510-13523.

141. Huang X, Wang J, Liu H, Lan T, Ren J. Quantum dot-based FRET for sensitive determination of hydrogen peroxide and glucose using tyramide reaction. Talanta. 2013;106:79-84.

142. Cao X, Lin W, Zheng K, He L. A near-infrared fluorescent turn-on probe for fluorescence imaging of hydrogen sulfide in living cells based on thiolysis of dinitrophenyl ether. Chem Comm. 2012;48(85): 10529-10531.
143. Yuan L, Lin W, Yang Y, Chen H. A unique class of near-infrared functional fluorescent dyes with carboxylic-acid-modulated fluorescence ON/OFF switching: rational design, synthesis, optical properties, theoretical calculations, and applications for fluorescence imaging in living animals. J Am Chem Soc. 2012;134(2):1200-1211.

144. Karton-Lifshin N, Albertazzi L, Bendikov M, Baran PS, Shabat D. "Donor-two-acceptor" dye design: a distinct gateway to NIR fluorescence. J Am Chem Soc. 2012;134(50):20412-20420.

145. Kisin-Finfer E, Shabat D. New repertoire of 'donor-two-acceptor' NIR fluorogenic dyes. Bioorg Med Chem. 2013;21(12):3602-3608.

146. Menéndez GO, Pichel ME, Spagnuolo CC, Jares-Erijman EA. NIR fluorescent biotinylated cyanine dye: optical properties and combination with quantum dots as a potential sensing device. Photochem Photobiol Sci. 2013;12(2):236-240.

147. van der Vorst JR, Hutteman M, Mieog JS, et al. Near-infrared fluorescence imaging of liver metastases in rats using indocyanine green. J Surg Res. 2012;174(2):266-271.

148. Morita Y, Sakaguchi T, Unno N, et al. Detection of hepatocellular carcinomas with near-infrared fluorescence imaging using indocyanine green: its usefulness and limitation. Int J Clin Oncol. 2013;18(2): 232-241.

149. Sherwinter DA. Transanal near-infrared imaging of colorectal anastomotic perfusion. Surg Laparosc Endosc Percutan Tech. 2012;22(5):433-436

150. Li J, Chen K, Liu H, et al. Activatable near-infrared fluorescent probe for in vivo imaging of fibroblast activation protein-alpha. Bioconjug Chem. 2012;23(8):1704-1711.

151. Sevick-Muraca EM. Translation of near-infrared fluorescence imaging technologies: emerging clinical applications. Ann Rev Med. 2012;63: 217-231.

152. Aldrich MB, Davies-Venn C, Angermiller B, et al. Concentration of indocyanine green does not significantly influence lymphatic function as assessed by near-infrared imaging. Lymphat Res Biol. 2012;10(1): 20-24.

153. Weiler M, Kassis T, Dixon JB. Sensitivity analysis of near-infrared functional lymphatic imaging. J Biomed Opt. 2012;17(6):066019.

154. Li P, Sun P, Yang W, Zhang X. Real-time mapping of rat stomach lymph nodes by quantum dots. Scand J Gastroenterol. 2012;47(4): 454-460.

155. Proulx ST, Luciani P, Christiansen A, et al. Use of a PEG-conjugated bright near-infrared dye for functional imaging of rerouting of tumor lymphatic drainage after sentinel lymph node metastasis. Biomaterials. 2013;34(21):5128-5137.

156. Weiler M, Dixon JB. Differential transport function of lymphatic vessels in the rat tail model and the long-term effects of Indocyanine Green as assessed with near-infrared imaging. Front Physiol. 2013;4:215.

157. Jeong HS, Lee CM, Cheong SJ, et al. The effect of mannosylation of liposome-encapsulated indocyanine green on imaging of sentinel lymph node. J Liposome Res. 2013;23(4):291-297.

158. Noh YW, Kong SH, Choi DY, et al. Near-infrared emitting polymer nanogels for efficient sentinel lymph node mapping. ACS Nano. 2012;6(9):7820-7831.

159. Maus EA, Tan IC, Rasmussen JC, et al. Near-infrared fluorescence imaging of lymphatics in head and neck lymphedema. Head Neck. 2012;34(3):448-453.

160. van der Vorst JR, Schaafsma BE, Verbeek FP, et al. Near-infrared fluorescence sentinel lymph node mapping of the oral cavity in head and neck cancer patients. Oral Oncol. 2013;49(1):15-19.

161. Gilmore DM, Khullar OV, Gioux S, et al. Effective low-dose escalation of indocyanine green for near-infrared fluorescent sentinel lymph node mapping in melanoma. Ann Surg Oncol. 2013;20(7):2357-2363.

162. van der Vorst JR, Schaafsma BE, Verbeek FP, et al. Dose optimization for near-infrared fluorescence sentinel lymph node mapping in patients with melanoma. Br J Dermatol. 2013;168(1):93-98.

163. Stoffels I, von der Stuck H, Boy C, et al. Indocyanine green fluorescence-guided sentinel lymph node biopsy in dermato-oncology. J Dtsch Dermatol Ges. 2012;10(1):51-57. 
164. Schaafsma BE, Verbeek FP, van der Vorst JR, et al. Ex vivo sentinel node mapping in colon cancer combining blue dye staining and fluorescence imaging. J Surg Res. 2013;183(1):253-257.

165. Schaafsma BE, Verbeek FP, Rietbergen DD, et al. Clinical trial of combined radio- and fluorescence-guided sentinel lymph node biopsy in breast cancer. Br J Surg. 2013;100(8):1037-1044.

166. Polom K, Murawa D, Nowaczyk P, Rho YS, Murawa P. Breast cancer sentinel lymph node mapping using near infrared guided indocyanine green and indocyanine green - human serum albumin in comparison with gamma emitting radioactive colloid tracer. Eur J Surg Oncol. 2012;38(2):137-142.

167. Schaafsma BE, Verbeek FP, Peters AA, et al. Near-infrared fluorescence sentinel lymph node biopsy in vulvar cancer: a randomised comparison of lymphatic tracers. BJOG. 2013;120(6):758-764.

168. Rossi EC, Ivanova A, Boggess JF. Robotically assisted fluorescenceguided lymph node mapping with ICG for gynecologic malignancies: a feasibility study. Gynecol Oncol. 2012;124(1):78-82.

169. Cahill RA, Anderson M, Wang LM, Lindsey I, Cunningham C, Mortensen NJ. Near-infrared (NIR) laparoscopy for intraoperative lymphatic road-mapping and sentinel node identification during definitive surgical resection of early-stage colorectal neoplasia. Surg Endosc. 2012;26(1):197-204.

170. Mieszawska AJ, Kim Y, Gianella A, et al. Synthesis of polymer-lipid nanoparticles for image-guided delivery of dual modality therapy. Bioconjug Chem. 2013;24(9):1429-1434.

171. Li M, Yang X, Ren J, Qu K, Qu X. Using graphene oxide high near-infrared absorbance for photothermal treatment of Alzheimer's disease. Adv Mater. 2012;24(13):1722-1728.

172. Hu SH, Chen YW, Hung WT, Chen IW, Chen SY. Quantum-dottagged reduced graphene oxide nanocomposites for bright fluorescence bioimaging and photothermal therapy monitored in situ. Adv Mater. 2012;24(13):1748-1754.

173. Radzi R, Osaki T, Tsuka T, et al. Photodynamic hyperthermal therapy with indocyanine green (ICG) induces apoptosis and cell cycle arrest in B16F10 murine melanoma cells. J Vet Med Sci. 2012;74(5): $545-551$.

174. Shafirstein G, Baumler W, Hennings LJ, et al. Indocyanine green enhanced near-infrared laser treatment of murine mammary carcinoma. Int J Cancer. 2012;130(5):1208-1215.

175. Tang Y, McGoron AJ. Increasing the rate of heating: a potential therapeutic approach for achieving synergistic tumour killing in combined hyperthermia and chemotherapy. Int J Hyperthermia. 2013;29(2): $145-155$.

176. Wu L, Fang S, Shi S, Deng J, Liu B, Cai L. Hybrid polypeptide micelles loading indocyanine green for tumor imaging and photothermal effect study. Biomacromolecules. 2013;14(9):3027-3033.

177. Fernandez-Fernandez A, Manchanda R, Lei T, et al. Comparative study of the optical and heat generation properties of IR820 and indocyanine green. Mol Imaging. 2012;11(2):99-113.

178. Lim CK, Shin J, Lee YD, et al. Phthalocyanine-aggregated polymeric nanoparticles as tumor-homing near-infrared absorbers for photothermal therapy of cancer. Theranostics. 2012;2(9):871-879.

179. Gutwein LG, Singh AK, Hahn MA, et al. Fractionated photothermal antitumor therapy with multidye nanoparticles. Int J Nanomed. 2012;7: 351-357.

180. Yue C, Liu P, Zheng M, et al. IR-780 dye loaded tumor targeting theranostic nanoparticles for NIR imaging and photothermal therapy. Biomaterials. 2013;34(28):6853-6861.

181. Qian J, Wang D, Cai F, Zhan Q, Wang Y, He S. Photosensitizer encapsulated organically modified silica nanoparticles for direct two-photon photodynamic therapy and in vivo functional imaging. Biomaterials. 2012;33(19):4851-4860.

182. Gupta A, Wang S, Pera P, et al. Multifunctional nanoplatforms for fluorescence imaging and photodynamic therapy developed by post-loading photosensitizer and fluorophore to polyacrylamide nanoparticles. Nanomedicine. 2012;8(6):941-950.
183. Nakajima T, Sano K, Mitsunaga M, Choyke PL, Kobayashi H. Real-time monitoring of in vivo acute necrotic cancer cell death induced by near infrared photoimmunotherapy using fluorescence lifetime imaging. Cancer Res. 2012;72(18):4622-4628.

184. Mitsunaga M, Nakajima T, Sano K, Kramer-Marek G, Choyke PL, Kobayashi H. Immediate in vivo target-specific cancer cell death after near infrared photoimmunotherapy. BMC Cancer. 2012;12:345.

185. Mitsunaga M, Nakajima T, Sano K, Choyke PL, Kobayashi H. Near-infrared theranostic photoimmunotherapy (PIT): repeated exposure of light enhances the effect of immunoconjugate. Bioconjug Chem. 2012;23(3):604-609.

186. Zandberg WF, Bakhtiari AB, Erno Z, et al. Photothermal release of small molecules from gold nanoparticles in live cells. Nanomedicine. 2012;8(6):908-915.

187. Ganesh S, Iyer AK, Gattacceca F, Morrissey DV, Amiji MM. In vivo biodistribution of siRNA and cisplatin administered using CD44-targeted hyaluronic acid nanoparticles. J Control Release. 2013;172(3): 699-706.

188. Cheng Y, Yu S, Zhen X, Wang X, Wu W, Jiang X. Alginic acid nanoparticles prepared through counterion complexation method as a drug delivery system. ACS Appl Mater Interfaces. 2012;4(10): 5325-5332.

189. Khullar OV, Griset AP, Gibbs-Strauss SL, et al. Nanoparticle migration and delivery of Paclitaxel to regional lymph nodes in a large animal model. J Am Coll Surg. 2012;214(3):328-337.

190. Bagby TR, Duan S, Cai S, et al. Lymphatic trafficking kinetics and near-infrared imaging using star polymer architectures with controlled anionic character. Eur J Pharm Sci. 2012;47(1):287-294.

191. Cao J, Huang S, Chen Y, et al. Near-infrared light-triggered micelles for fast controlled drug release in deep tissue. Biomaterials. 2013;34(26):6272-6283.

192. Turner DC, Moshkelani D, Shemesh CS, Luc D, Zhang H. Nearinfrared image-guided delivery and controlled release using optimized thermosensitive liposomes. Pharm Res. 2012;29(8):2092-2103.

193. Xu M, Rettig MP, Sudlow G, et al. Preclinical evaluation of Mab CC188 for ovarian cancer imaging. Int J Cancer. 2012;131(6):1351-1359.

194. Day KE, Beck LN, Heath CH, Huang CC, Zinn KR, Rosenthal EL. Identification of the optimal therapeutic antibody for fluorescent imaging of cutaneous squamous cell carcinoma. Cancer Biol Ther. 2013;14(3):271-277.

195. Day KE, Beck LN, Deep NL, Kovar J, Zinn KR, Rosenthal EL. Fluorescently labeled therapeutic antibodies for detection of microscopic melanoma. Laryngoscope. 2013;123(11):2681-2689.

196. Gong H, Kovar JL, Baker B, et al. Near-infrared fluorescence imaging of mammalian cells and xenograft tumors with SNAP-tag. PloS one. 2012;7(3):e34003.

197. Kim SH, Park G, Hyun H, et al. Near-infrared lipophilic fluorophores for tracing tissue growth. Biomed Mater. 2013;8(1):014110.

198. Merian J, Gravier J, Navarro F, Texier I. Fluorescent nanoprobes dedicated to in vivo imaging: from preclinical validations to clinical translation. Molecules. 2012;17(5):5564-5591.

199. Du Y, An S, Liu L, et al. Serial non-invasive monitoring of renal disease following immune-mediated injury using near-infrared optical imaging. PloS one. 2012;7(9):e43941.

200. Cao J, Wan S, Tian J, et al. Fast clearing RGD-based near-infrared fluorescent probes for in vivo tumor diagnosis. Contrast Media Mol Imaging. 2012;7(4):390-402.

201. Keereweer S, Mol IM, Kerrebijn JD, et al. Targeting integrins and enhanced permeability and retention (EPR) effect for optical imaging of oral cancer. J Surg Oncol. 2012;105(7):714-718.

202. Iftimia N, Iyer AK, Hammer DX, et al. Fluorescence-guided optical coherence tomography imaging for colon cancer screening: a preliminary mouse study. Biomed Opt Express. 2012;3(1):178-191.

203. Zhang L, Wang K, Zhao F, et al. Near infrared imaging of EGFR of oral squamous cell carcinoma in mice administered arsenic trioxide. PloS one. 2012;7(9):e46255. 
204. Agnes RS, Broome AM, Wang J, Verma A, Lavik K, Basilion JP. An optical probe for noninvasive molecular imaging of orthotopic brain tumors overexpressing epidermal growth factor receptor. Mol Cancer Ther. 2012;11(10):2202-2211.

205. Keereweer S, Mol IM, Vahrmeijer AL, et al. Dual wavelength tumor targeting for detection of hypopharyngeal cancer using near-infrared optical imaging in an animal model. Int J Cancer. 2012;131(7): 1633-1640.

206. Lee CM, Jang D, Cheong SJ, et al. Optical imaging of MMP expression and cancer progression in an inflammation-induced colon cancer model. Int J Cancer. 2012;131(8):1846-1853.
207. Chen Y, Pullambhatla M, Banerjee SR, et al. Synthesis and biological evaluation of low molecular weight fluorescent imaging agents for the prostate-specific membrane antigen. Bioconjug Chem. 2012;23(12): 2377-2385.

208. Zhou J, Tsai YT, Weng H, et al. Real-time detection of implantassociated neutrophil responses using a formyl peptide receptortargeting NIR nanoprobe. Int J Nanomed. 2012;7:2057-2068.

209. Pauli J, Licha K, Berkemeyer J, et al. New fluorescent labels with tunable hydrophilicity for the rational design of bright optical probes for molecular imaging. Bioconjug Chem. 2013;24(7):1174-1185.
International Journal of Nanomedicine

\section{Publish your work in this journal}

The International Journal of Nanomedicine is an international, peerreviewed journal focusing on the application of nanotechnology in diagnostics, therapeutics, and drug delivery systems throughout the biomedical field. This journal is indexed on PubMed Central,

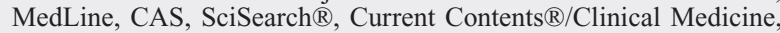

\section{Dovepress}

Journal Citation Reports/Science Edition, EMBase, Scopus and the Elsevier Bibliographic databases. The manuscript management system is completely online and includes a very quick and fair peer-review system, which is all easy to use. Visit http://www.dovepress.com/ testimonials.php to read real quotes from published authors.

Submit your manuscript here: http://www.dovepress.com/international-journal-of-nanomedicine-journal 